\title{
The effects of ultrasound exposure on P-glycoprotein-mediated multidrug resistance in vitro and in vivo
}

Chixiong Huang ${ }^{1 \dagger}$, Senlin Huang ${ }^{\dagger}$, Hairui $\mathrm{Li}^{1}$, Xinzhong $\mathrm{Li}^{1}$, Bing $\mathrm{Li}^{1}$, Lintao Zhong ${ }^{1}$, Junfeng Wang ${ }^{2}$, Meishen Zou', Xiang $\mathrm{He}^{1}$, Hao Zheng ${ }^{1}$, Xiaoyun $\mathrm{Si}^{1}$, Wangjun Liao ${ }^{3}$, Yulin Liao ${ }^{1}$, Li Yang ${ }^{4^{*}}$ and Jianping Bin ${ }^{1^{*}}$

\begin{abstract}
Background: Multidrug resistance (MDR) is often responsible for the failure of chemotherapy treatment, and current strategies for cancer MDR are not adequately satisfying as to their efficacy and safety. In this study, we sought to determine the anti-MDR effects of ultrasound (US) irradiation and its underlying mechanisms against drugresistance.
\end{abstract}

Methods: MDR variant MCF-7/ADR cell lines and endothelial cell lines were used to determine the appropriate ultrasound intensity for in vitro experiments. MCF-7/ADR cell and HEPG2/ADM cells were used to assess the anti-MDR effect of US irradiation. Intracellular adriamycin (ADM) accumulation, Cell viability, cell proliferation and cell apoptosis were evaluated after ADM + US treatment or ADM treatment alone. MCF-7/ADR xenograft mice were used to investigate the appropriate ultrasound intensity for in vivo experiments and its effect on the long-term prognosis. Underlining mechanisms by which ultrasound exposure reversing MDR phenotype were investigated both in vitro and in vivo.

Results: Combination of ADM and $0.74 \mathrm{~W} / \mathrm{cm}^{2}$ US irradiation enhanced ADM intracellular concentration and nuclear accumulation in MCF-7/ADR and HEPG2/ADM cells, compared to those treated with ADM alone. Enhanced cellular ADM uptake and nuclei localization was associated with increased cytotoxicity of ADM to ADM-resistant cells, lower ADM-resistant cell viability and proliferative cell ratio, and higher apoptotic cell ratio. More importantly, US exposure increased the effectiveness of ADM to inhibit tumor growth in MCF-7/ADR xenograft mice. Mechanistically, US exposure promoted ADM accumulation in MDR cells mainly through downregulation of P-glycoprotein (P-gp), which is dependent on US-induced intracellular reactive oxygen species (ROS) production. US-induced oxidative stress promoted miR-200c-3p and miR-34a-3p expression by forming miR-200c/34a/ZEB1 double-negative feedback loop. Finally, US-induced miR-200c/34a overexpression decreased P-gp expression and reversed MDR phenotype.

Conclusion: US irradiation could reverse MDR phenotype by activating ROS-ZEB1-miR200c/34a-P-gp signal pathway. Our findings offer a new and promising strategy for sensitizing cells to combat MDR and to improve the therapeutic index of chemotherapy.

Keywords: Ultrasound, Multidrug resistance, P-glycoprotein, Reactive oxygen species

\footnotetext{
*Correspondence: nfyang@126.com; jianpingbin@126.com; jianpingbin@hotmail.com

${ }^{+}$Chixiong Huang and Senlin Huang contributed equally to this work.

${ }^{4}$ Department of Pharmacy, Nanfang Hospital, Southern Medical University, Guangzhou, People's Republic of China

${ }^{1}$ State Key Laboratory of Organ Failure Research, Department of Cardiology,

Nanfang Hospital, Southern Medical University, 1838 Guangzhou Avenue

North, Guangzhou 510515, China

Full list of author information is available at the end of the article
}

(c) The Author(s). 2018 Open Access This article is distributed under the terms of the Creative Commons Attribution 4.0 International License (http://creativecommons.org/licenses/by/4.0/), which permits unrestricted use, distribution, and reproduction in any medium, provided you give appropriate credit to the original author(s) and the source, provide a link to the Creative Commons license, and indicate if changes were made. The Creative Commons Public Domain Dedication waiver (http://creativecommons.org/publicdomain/zero/1.0/) applies to the data made available in this article, unless otherwise stated. 


\section{Background}

Chemotherapy is one of the most effective treatments for malignant tumors. The progressive inducement to multidrug resistance (MDR), however, is often responsible for the final failure of chemotherapy treatment, and it is believed to be one of the leading reasons making cancers incurable $[1,2]$. It is estimated that nearly $90 \%$ of the cancer patients with metastasis fail in their treatment due to developed MDR [3]. Although multifactorial in mechanism, enhanced drug efflux mediated by membranal P-glycoprotein (P-gp) is believed to be one major cause of cancer MDR [4, 5]. P-gp, also known as ATP binding cassette subfamily $B$ member 1 (ABCB-1) or MDR1, is a member of the ATP-binding cassette transporter family that prevents anticancer drugs from intracellular accumulation to a therapeutic level by extruding these drugs across the cytomembrane [6, 7]. Cancer drugs such as adriamycin (ADM), paclitaxel, daunorubicin, and epirubicin are common substrates of P-gp. Evidence from preclinical models revealed that overexpression of P-gp led to MDR resistance against multiple cancer drugs; in cancer patients, P-gp upregulation was related to suboptimal treatment response and poor long-term prognosis [8]. Therefore, the inhibition of P-gp is one of the most extensively studied strategies for MDR reversal.

Unfortunately, current solutions for P-gp mediated MDR are not sufficiently effective or safe/far from being satisfactory. Although three distinct generations of P-gp inhibitors have been produced in the past 30 years, a clinically serviceable modulator has yet to be developed [9]. A major deficiency of P-gp inhibitors is their indiscriminate distribution among organs and nonspecific action on P-gp [10]. Since P-gp has shown a protective role in several important organs and tissues, such as indigestive system, the blood-testis barrier, the blood-brain barrier, and membranes of many types of stem cells [11], systemic administration of P-gp inhibitors may lead to systemic toxicity. To overcome this toxicity, researchers are investigating the use of a nanoparticle-mediated specific drug delivery system to tumor tissues to restrict irrelevant P-gp inhibition [12]. Recent studies have also explored the efficacy of local prevention of the biosynthesis of P-gp in tumors using RNAi or miRNAs delivered by viral vectors [13-15]. These efforts are similarly hindered, however, by challenges such as accurate viral location, expression efficiency, stability, and administration safety in vivo. Taken together, these applications are still problematic in their translation from experiments to clinic.

Ultrasound (US), a form of mechanical energy, has been shown the ability to open cell membranes, enhancing delivery of drugs, proteins, and genes through what is known as sonoporation effect [16]. Intriguingly, recent studies have revealed that US exposure significantly repressed P-gp expression [17-21], suggesting that US exposure may facilitate intracellular accumulation of chemotherapy drugs in MDR cancer cells. Because the mechanical energy of US can be focused on an area as tiny as at millimeter level, US exposure could accurately restrict the sonoporation effect or P-gp inhibition to an appointed target, thus avoiding toxicity in nontarget organs. Therefore, US exposure may be a promising approach in the treatment of MDR for its ability to locally increase anticancer agent concentrations in MDR cancer tissues. However, the efficacy and feasibility of this new approach in sensitizing drug-resistant cells to chemotherapy treatment and improving long-term outcomes has not been evaluated in vivo. Moreover, the underlying mechanism by which US decreased P-gp expression is still largely unknown.

In the current study, our observations demonstrated that US exposure increased cytotoxicity of ADM to ADM-resistant cells both in vitro and in vivo. Further, we linked the anti-MDR effect of US exposure to downregulation of P-gp. US exposure promoted reactive oxygen species (ROS) generation and triggered a doublenegative feedback loop involving ZEB1 and miR-200c/ 34a. US-induced miR-200c/34a directly or indirectly inhibited P-gp expression and reversed MDR phenotype.

\section{Methods}

\section{Cell culture}

We purchased human umbilical vein endothelial cell (HUVEC) lines, MCF-7, HEPG2, MCF-7/ADR, and HEPG2/ADM cell lines from Geneseed (Guangzhou, China). The cells were cultured in RPMI 1640 (Gibco, USA) containing $10 \%$ fetal bovine serum (FBS; Gibco Laboratories, Gaithersburg, MD, USA), $100 \mathrm{U} / \mathrm{ml}$ penicillin and $100 \mu \mathrm{g} / \mathrm{ml}$ streptomycin (Gibco), and $2 \mathrm{mM} \mathrm{L-glu-}$ tamine. To maintain the drug-resistant phenotype, we cultured MCF-7/ADR and HEPG2/ADM cells in the presence of $1 \mu \mathrm{g} / \mathrm{ml}$ ADM (MedChemExpress, Monmouth Junction, NJ, USA) and passaged the cells for 1 week in a drug-free medium before beginning the experiment. We maintained the cells in a humidified incubator in the presence of $5 \% \mathrm{CO} 2$.

\section{ADM uptake and intracellular distribution in cells}

To detect ADM accumulation in cells, HUVEC, MCF-7, HEPG2, MCF-7/ADR or HEPG2/ADM cells were at $2 \times 10^{\wedge} 6$ cells/well in 6-well plates and incubated overnight. Unless otherwise stated, cells were immediately subjected to US irradiation after addition of ADM. After that, cells were incubated for another $24 \mathrm{~h}$. Then, we rinsed the cells to remove un-internalized ADM. To quantitatively determine cellular ADM concentrations, the treated cells were collected and lysed with RIPA cell lysis buffer (BestBio, Shanghai, China), and the ADM concentration in the cell lysates was detected using a 
microplate reader (Synergy $^{\text {tw }}$ 4, BioTek Instruments, Winooski, VT, USA) at excitation and emission wavelengths of $485 / 550 \mathrm{~nm}$ [22]. We normalized the results to total cellular protein content, which we determined using the BCA protein assay kit (BestBio, China).

To determine the intracellular distribution of ADM, the cells were seeded in a confocal culture dish at a concentration of $5 \times 10^{\wedge} 5$ cells. Following treatment, we rinsed the cells three times with PBS and fixed them with a $4 \%$ paraformaldehyde solution for $30 \mathrm{~min}$ at room temperature. We then rinsed the cells with PBS three times for $5 \mathrm{~min}$ each time, and stained the cells with DAPI for $5 \mathrm{~min}$. We then washed the cells three times with PBS to remove extracellular DAPI and examined the cells with a confocal laser scanning microscope (LSM 880 with Airyscan, Carl Zeiss, Germany) to view localized ADM in the cells at excitation and emission wave lengths of $485 / 550 \mathrm{~nm}$.

\section{Cytotoxicity assay}

We seeded HUVEC, MCF-7, HEPG2, MCF-7/ADR or HEPG2/ADM cells at $10^{6}$ cells/well in 6-well plates and incubated the cells for $24 \mathrm{~h}$. Cells were incubated with ADM or other modulators. Then, US irradiation was performed as stated above. After $24 \mathrm{~h}$ treatment, we determined cell viability via MTT assay as described previously [23]. We measured absorbance at $450 \mathrm{~nm}$ using a multimode plate reader (Synergy $^{\text {mix }} 4$, BioTek Instruments). Bliss model was used to calculate the IC50 of ADM as described previously [24].

\section{Determination of optimal US parameters in vitro}

A pulsed therapeutic ultrasound device with a KHT-017 transducer (DCT-700, Shenzhen Well.D Medical Electronic, Shenzhen, China) was used for ultrasound stimulation. As described previously [25], the sterilized transducer was fixed on a steel stand with scale to maintain a distance of $10 \mathrm{~mm}$ between the transducer and cultured cell in monolayers (Additional file 1: Figure S1). The transducer was operated at a frequency of $1 \mathrm{MHz}$ with a pulse repetition frequency of $10 \mathrm{~Hz}$ and a duty cycle of $20 \%$ for 5 min.

To determine the optimal acoustic intensity of US in vitro, we seeded MCF-7/ADR at a concentration of $10^{\wedge} 6$ cells in 6-well plates and incubated the cells for $24 \mathrm{~h}$ to allow for adhesion. ADM was added to medium before application of US under differentiated peak negative acoustic intensities $(0,0.09,0.21,0.40,0.74$, and $1.22 \mathrm{~W} /$ $\mathrm{cm}^{2}$ ). Then cells were incubated for another $24 \mathrm{~h}$. We detected cell viability via MTT assay. We detected ADM fluorescence and intracellular distribution as described above.

\section{Flow cytometry analysis}

MCF-7/ADR or HEPG2/ADM cells were treated with $\mathrm{ADM}$ or ADM + US. At $24 \mathrm{~h}$ after treatment, we determined cell apoptosis using flow cytometry (LSRFortessa $^{\text {Tax }}$, BD Biosciences, San Jose, CA, USA) via PI and FITC (BestBio) staining. Cells were collected and washed three times in ice-cold PBS. Cells were resuspended in $400 \mu \mathrm{l}$ of Annexin $\mathrm{V}$ which contained $5 \mu \mathrm{l}$ Annexin V-FITC. Following $15 \mathrm{~min}$ of incubation at $4{ }^{\circ} \mathrm{C}$, we added $10 \mu \mathrm{l}$ of PI to the suspension and incubated for 5 min. We detected apoptosis using flow cytometry (BD LSRFortessa X-20).

\section{Scanning Electron microscopy}

We observed structural changes in cells following exposure to the optimum US conditions (immediately, $30 \mathrm{~min}$, $60 \mathrm{~min}, 24 \mathrm{~h}$ ), as compared with cells without US irradiation, using SEM. We seeded HUVEC, MCF-7, HEPG2, MCF-7/ADR or HEPG2/ADM cells at a concentration of $10^{\wedge} 6$ cells in 6 -well plates and incubated the cells for $24 \mathrm{~h}$ to allow for adhesion. We performed US with the determined optimal parameters as described above. Following treatment, we washed the cells twice with PBS and resuspended the cells in PBS. Cell suspensions were dropped on a cover glass with a gold-plated membrane for $30 \mathrm{~min}$. The cells were fixed with $4 \%$ formalin for $2-3 \mathrm{~min}$ and then were washed with triple PBS for $10 \mathrm{~min}$. The cells were then fixed with $1 \%$ osmic acid for 20-30 min and then were washed with ice-cold distilled water three times. Then the cells were soaked in $2 \%$ tannin at $4{ }^{\circ} \mathrm{C}$ overnight, dehydrated using graded ethanol, and lyophilized using tertiary butyl alcohol overnight. Finally, the cells were coated using a vacuum spray-plating instrument (Hitachi $\mathrm{H}-7500$ ) and images were captured.

\section{Preparation of MCF-7/ADR xenograft nude mice}

We purchased 60 female BALB/C-nude mice aged 4 weeks from Guangdong Medical Laboratory Animal Center (GDMLAC). Following subcutaneous injection of $10^{\wedge} 7 \mathrm{MCF}-7 / \mathrm{ADR}$ cells into the right oxter, we observed nude mice for 4 weeks, or until solid tumor growth reached $0.5-1.0 \mathrm{~cm}^{3}$. We calculated tumor volume $(\mathrm{V})$ as follows: $\pi \times\left(\mathrm{L} \times \mathrm{W}^{2}\right) / 6$ (L: length, W: width) [26].

\section{Determination of optimal US parameters in vivo}

To explore an appropriate level of ultrasound intensity, 24 MCF-7/ADR xenograft nude mice were randomized into six group treated with ADM $(8 \mathrm{mg} / \mathrm{kg})$ intravenously (i.v.) + US exposure of differentiated peak negative acoustic intensities $(0.09,0.21,0.40,0.74$, and $1.22 \mathrm{~W} /$ $\mathrm{cm}^{2}$ ) every 2 days (q2d). The US transducer was placed in contact with skin overlying the xenograft. The other parameters of US stimulation in vivo were the same as that used in vitro. After 7 days, the mice were sacrificed. 
The tumor tissues and surrounding muscle tissues were isolated for further experiments. Tissue apoptosis was detected by terminal deoxynucleotidyl transferase dUTP nick end labeling (TUNEL) staining per the manufacturer's protocol (Roche-Diagnostics, Indianapolis, IN, USA). We detected the apoptotic cells (stained red) using confocal microscopy, ADM fluorescence of tumor tissues, and surrounding muscle tissue using microplate readers. The intensity of US exposure, which enhanced intracellular ADM concentrations without inducing apoptosis in surrounding muscle tissues, were used for further studies.

\section{Reversal of MDR in vivo}

To assess the synergistical effect of US exposure and ADM treatment in vivo, $12 \mathrm{MCF}-7 / \mathrm{ADR}$ xenograft nude mice were randomly subjected into treatment groups of three, including groups receiving ADM alone $(8 \mathrm{mg} / \mathrm{kg}$, i.v. $\mathrm{q} 2 \mathrm{~d})$, both ADM (8 $\mathrm{mg} / \mathrm{kg}$, i.v. $)+$ US exposure ( $2 \mathrm{~min}$ ), q3d. The drug dosage was modulated according to the animal's weight every 6 days. Tumor growth was monitored since the first day of treatment and the tumor volume was measured every 6 days. Twenty-four days later, the mice were sacrificed. The excised tumor tissues were used for further experiments.

\section{Immunohistochemical staining}

Tumor tissues were fixed with 4\% paraformaldehyde and paraffin-embedded. De-paraffinized sections were rehydrated through a graded alcohol series. Sections were boiled in antigen retrieval solution $(10 \mathrm{mM}$ sodium citrate, $0.05 \%$ Tween-20, $\mathrm{pH} 6.0$ ) for $20 \mathrm{~min}$ and then cooled for $30 \mathrm{~min}$. After blocking with $4 \%$ normal goat serum, the sections were incubated overnight with P-glycoprotein mAb (ab170904, Abcam), ZEB1 mAb (21544-1-AP, ProteinTech). The expression of P-gp or ZEB1 was assessed as described previously [27].

\section{Immunofluorescence staining}

Tumor tissues were collected and embedded in tissue-freezing medium. Cultured cells were fixed in $4 \%$ PFA (10 min). Then tissue sections and cultured cells were processed in the following methods. Samples were blocked with $4 \%$ normal goat serum and incubated for $3 \mathrm{~h}$ at room temperature with primary antibodies. The antibodies used are followed: anti-Ki67 antibody (1:200, 275R, Cell Marque), anti-P-gp antibody (ab170904, Abcam). When indicated, cells were stained using Click-it EdU imaging Kit (Life Technologies, \#C10638) to detect EdU incorporation, according to the manufacturer's instructions. Then, samples were washed with PBS and stained with secondary antibodies (Alexa Fluor 647, Abcam) for $1 \mathrm{~h}$ at room temperature. DAPI was used for the nuclear visualization. Image acquisition was performed using the confocal microscope (Carl Zeiss).

\section{ADM uptake and distribution in tumor tissue}

To detected ADM uptake in tissues, tissues were excised and lysed in lysis buffer after mice were sacrificed. After incubation for $30 \mathrm{~min}$ at room temperature and centrifugation at $14,000 \mathrm{rpm}$ for $10 \mathrm{~min}$, supernatant was transferred to a 96-well plate. ADM concentration in the supernatant was detected using microplate readers at excitation and emission wavelengths of $485 / 550 \mathrm{~nm}$. The results were normalized to total tissue protein content.

To detect ADM distribution in tissues, twenty-four hours after ADM administration, mice were sacrificed and tissues were collected and embedded in tissue-freezing medium. Tumor tissues were sectioned (10 $\mu \mathrm{m}$ thick) and imaged using a confocal microscope.

\section{TUNEL staining}

Tumor tissues were fixed with $4 \%$ paraformaldehyde and paraffin-embedded. Cultured cells were fixed in 4\% PFA (10 min). Then tissue sections and cultured cells were processed using In Situ Cell Death Detection Kit (Roche, Shanghai, China) to stain the apoptotic cells. Nuclei were stained with DAPI. We observed the tissue or cells using inverted fluorescence microscope (Olympus, Hamburg, Germany).

\section{Real-time polymerase chain reaction}

We isolated total RNA from cells or tissues by Trizol (Takara Bio, Inc., Shiga, Japan). $\beta$-catenin and U6 genes were used as gene and miRNA internal controls, respectively. Quantitative Polymerase chain reaction (Q-PCR) was performed by using the SYBR Green PCR Master Mix (TOYOBO Corp., Osaka-fu, Japan) in LightCycle480 (Roche, Germany). Cyclin conditions were as following: initial denaturation at $95{ }^{\circ} \mathrm{C}$ for $10 \mathrm{~min}$, followed by 40 cycles of $95{ }^{\circ} \mathrm{C}$ for $15 \mathrm{~s}, 60{ }^{\circ} \mathrm{C}$ for $15 \mathrm{~s}$, $72{ }^{\circ} \mathrm{C}$ for 32 s. Specific sense primers for $\mathrm{ABCB} 1$, miR-200c, miR-34a-3p, U6, ZEB1 and $\beta$-actin are shown in Additional file 2: Table S1.

\section{Western blot analysis}

We extracted total protein from treated cells or tissues using RIPA lysis buffer (BestBio) containing protease inhibitor cocktail Set I (BestBio). We separated proteins in $10 \%$ precast SDS-PAGE gels and transferred them onto a nitrocellulose membrane. The membrane was further incubated with primary P-glycoprotein mAb (1:100 dilution; ab170904, Abcam), ZEB1 mAb (1:100 dilution; 215441-AP, ProteinTech) or $\beta$-actin mAb (1:1000 dilution; Santa Cruz, CA, USA). We visualized the blots using an odyssey detection system (LI-COR Biosciences, Lincoln, NE, USA) and quantified protein abundance by ImageJ software. 
A

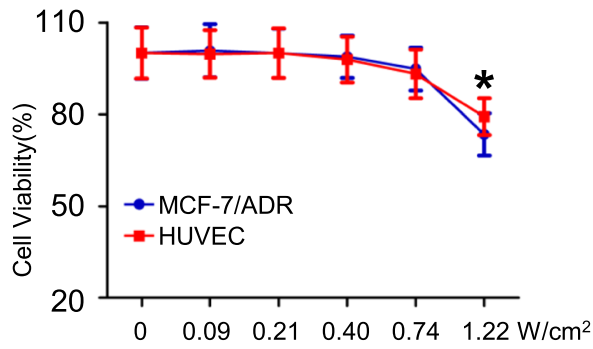

C

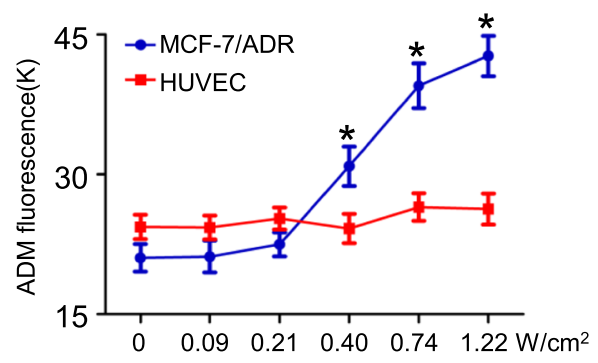

$\mathbf{E}$

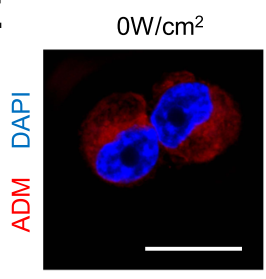

$\mathbf{F}$

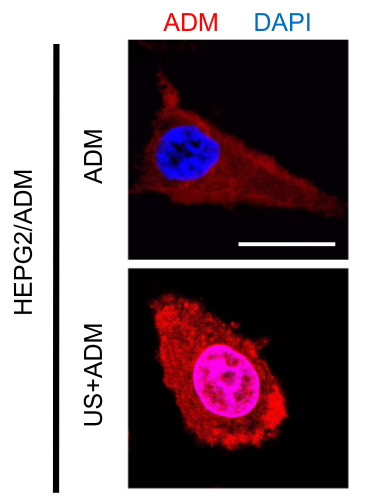

G

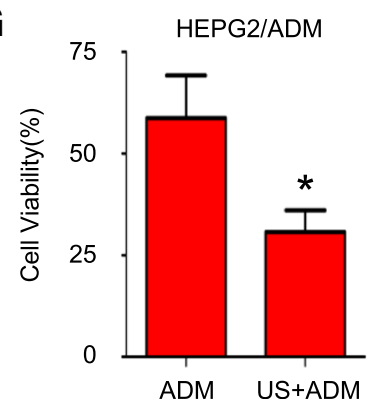

H
B

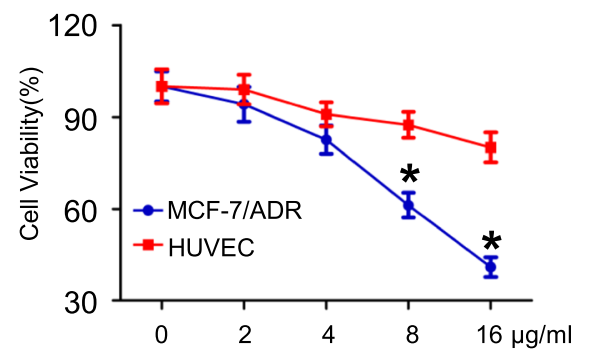

D
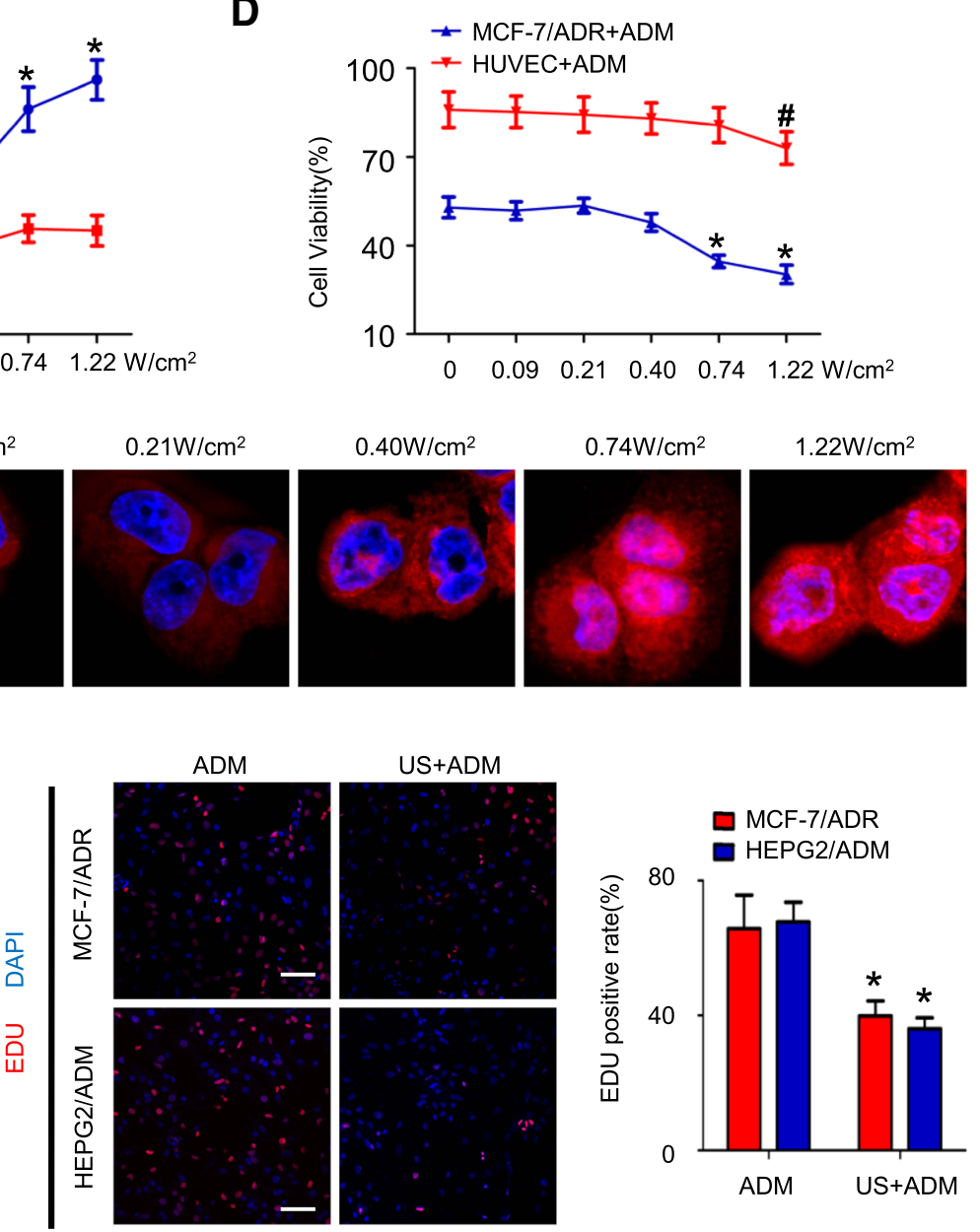

US+ADM
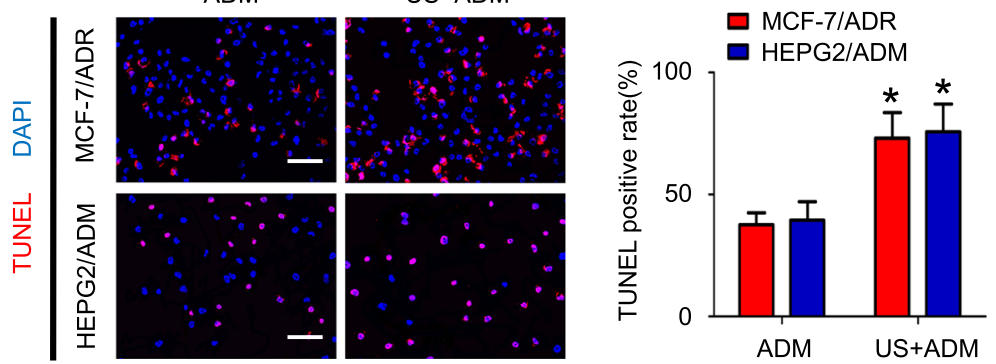

Fig. 1 (See legend on next page.) 
(See figure on previous page.)

Fig. 1 Exploration of optimal US parameters for reversing MDR in vitro. a Cell viability in MCF-7/ADR and HUVEC cells in $24 \mathrm{~h}$ after US exposure with different acoustic intensities; $N=3$; ${ }^{*} P<0.05$ vs. 0 W/ $/ \mathrm{cm}^{2}$ in MCF-7/ADR cells; (b) Cytotoxicity of ADM alone in the MCF-7/ADR and HUVEC cells; $\mathrm{N}=3$, ${ }^{*} P<0.05$ compared with HUVEC cells; (c) Intracellular ADM concentration in MCF-7/ADR and HUVEC cells in $24 \mathrm{~h}$ after US exposure with different acoustic intensities; $N=3 ;{ }^{*} P<0.05$ vs. 0 W/cm² in MCF-7/ADR cells; (d) Cytotoxicity of ADM in MCF-7/ADR and HUVEC cells post US+ADM treatment with different acoustic intensities; $N=3$; ${ }^{*} P<0.05 \mathrm{vs.} 0 \mathrm{~W} / \mathrm{cm}^{2}$ in MCF-7/ADR cells; data are represented as mean \pm s.d; (e) Images of intracellular ADM distribution in MCF-7/ADR cells post US+ADM treatment with different acoustic intensities $($ scale bar $=10 \mu \mathrm{m}) ;(\mathbf{f})$ Ultrasound acoustic intensity of $0.74 \mathrm{~W} / \mathrm{cm}^{2}$ enhanced intracellular ADM uptake and ADM nuclei localization in HEPG2/ADM cells; (g) Cytotoxicity of US+ADM or ADM alone in HEPG2/ADM cells; $N=3$; data are represented as mean \pm s.d; ${ }^{*} P<0.05$; (h) EDU staining and quantification of the proliferative cells in MCF-7/ADR and HEPG2/ADM cells post-treatment with US+ADM or ADM alone (scale bar $=50 \mu \mathrm{m}$ ); $\mathrm{N}=3$; data are represented as mean \pm s.d; ${ }^{*} P<0.05$; (i) TUNEL staining and quantification of the apoptotic cells in MCF-7/ADR and HEPG2/ADM cells post-treatment with US+ADM or ADM alone (scale bar $=50 \mu \mathrm{m}$ ); $\mathrm{N}=3$; data are represented as mean \pm s.d; ${ }^{*} P<0.05$

\section{Reactive oxygen species detection}

We detected the intracellular generation of ROS in cells using 2',7'-dichlorofluorescein-diacetate (DCFH-DA; Beyotime, Jiangsu, China). We seeded MCF-7/ADR or HEPG2/ADM cells at a concentration of $10^{\wedge} 6$ cells in 6-well plates and incubated the cells for $24 \mathrm{~h}$ to allow for adhesion. We added DCFH-DA at a final concentration of $10 \mu \mathrm{M}$ at $24 \mathrm{~h}$ post-treatment and incubated for another $20 \mathrm{~min}$ at $37{ }^{\circ} \mathrm{C}$. Cells were then lysed, and the lysates were centrifuged at $10,000 \times \mathrm{g}$ at $4{ }^{\circ} \mathrm{C}$ for $5 \mathrm{~min}$. We transferred the supernatant to black 96-well plates and measured the supernatant using a microplate reader at an excitation wavelength of $488 \mathrm{~nm}$ and emission wavelength of $525 \mathrm{~nm}$. Relative fluorescence units of the samples were calculated and normalized to the untreated cells. We also observed the cells by inverted fluorescence microscope (Olympus, Hamburg, Germany) after they were incubated for $20 \mathrm{~min}$ at $37^{\circ} \mathrm{C}$ in $10 \mu \mathrm{M}$ DCFH-DA.

\section{MiRNA mimics, miRNA inhibitors or siRNA transfection} MCF-7/ADR cells ( $1^{*} 10^{7}$ count) were seeded in 6-wells plates on the day before the transfection. MiR-200c mimic (100 pmol, RIBOBIO), miR-34a-3p mimic (100 pmol, RIBOBIO), mimic negative control (mimic-NC, 100 pmol, RIBOBIO), anta-miR-200c (100 pmol, RIBOBIO), antamiR-34a-3p (100 pmol, RIBOBIO), antagomir for negative control (anta-NC, 100 pmol, RIBOBIO), siRNA for ZEB1 (20 nM, RIBOMO) or siRNA for negative control (si-NC, $20 \mathrm{nM}$, RIBOMO) was used for the transfection of the cells, which was achieved by using Lipofectamine 2000 transfection reagent (Invirogen) according to manufacturers' protocol. At 24 after transfection, cells were treated with $\mathrm{ADM}$ or ADM + US as stated above.

\section{Luciferase assay}

The ZEB1 3'-UTR or ABCB1 3'-UTR sequence was PCR-amplified and cloned into the luciferase vector psiCHECK-2 (Saicheng Bio Co Ltd., China). The used primers for PCR amplification are shown in Additional file 2: Table S1. MCF-7/ADR cells were seeded in 24-well plates $24 \mathrm{~h}$ prior to transfection. For luciferase report assays, the miR-34a-3p or miR-200c mimic was co-transfected into MCF-7/ADR cells with constructed luciferase vectors. Firefly and Renilla luciferase activity was detected by the Dual Luciferase Reporter Assay system (Promega, Madison, WI).

\section{Chromatin immunoprecipitation assay}

The EpiQuik chromatin immunoprecipitation assay kit (EpiGentek, Brooklyn, NY) was applied according to the manufacturer's instructions. A $5 \mu \mathrm{g}$ portion of control IgG antibody or anti-ZEB1 antibody was used for immunoprecipitation. PCR was used to investigate enrichment of DNA fragments in the predicted ZEB1 binding sites in the miR-200c/34a promoters. The used primers are shown in Additional file 2: Table S1.

\section{Statistical analysis}

We displayed data as means plus or minus standard deviations. We calculated mean values from at least three experiments. We performed multiple comparisons using one-way analysis of variance (ANOVA) followed by Bonferroni correction. Differences were considered significant at $* P<0.05$. We performed statistical analyses using SPSS 13.0 (IBM SPSS, Chicago, IL, USA).

\section{Results}

US exposure enhances ADM-inducing cell killing in vitro

To determine the optimal US parameters which increase cytotoxicity of chemotherapy drugs for MDR cells but not for healthy cells, we observed the effect of ultrasound exposure on both MCF-7/ADR and HUVEC cells. We first studied whether US exposure itself inhibits cell growth in the MCF-7/ADR and HUVEC cells. Cell viability was more than $90 \%$ in both the MCF-7/ADR cells and HUVEC cells using ultrasound acoustic intensity $\leq 0.74 \mathrm{~W} / \mathrm{cm}^{2}$ exposure (Fig. 1a). Then, we examined MCF-7/ADR cells and HUVEC cells for their response to ADM treatment. The IC50 of ADM concentration for MCF-7/ADR cells is $12.19 \pm 1.65 \mu \mathrm{g} / \mathrm{ml}$ (Additional file 3: Figure S2A and Additional file 4: Table S2). MCF-7/ ADR cells are more sensitive to ADM compared with HUVEC cells $(P<0.05, \geq 8 \mu \mathrm{g} / \mathrm{ml}$ ADM; Fig. $1 \mathrm{~b})$. Their 


\section{B}

A $\mathrm{CON}$
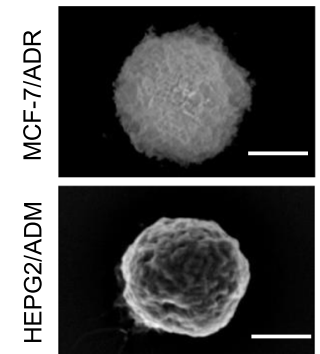

C
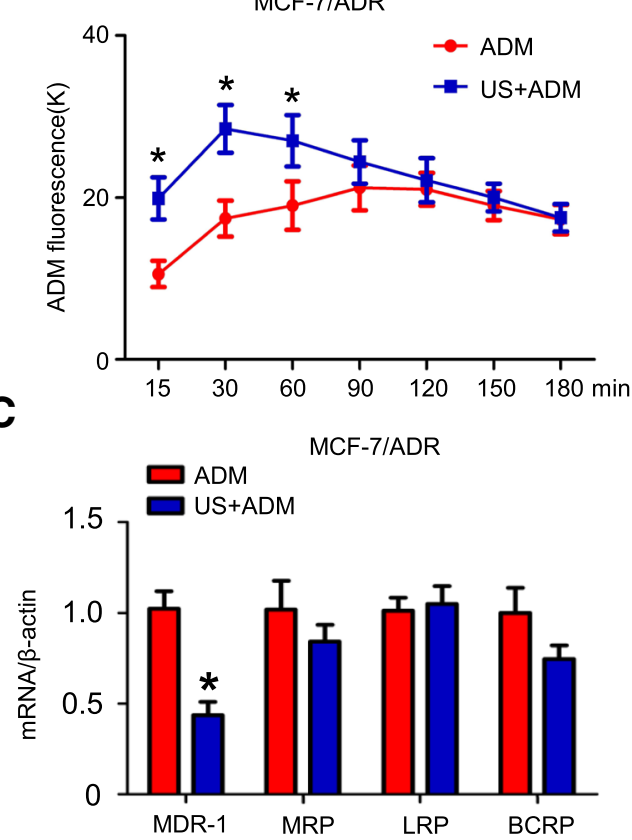

D

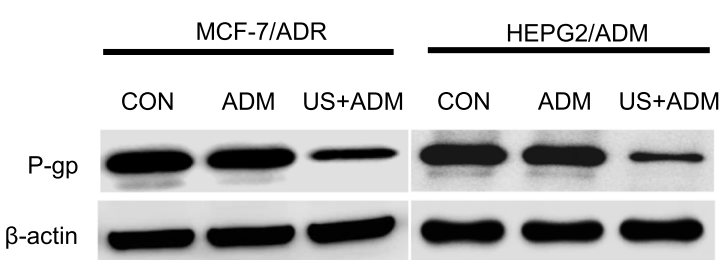

E

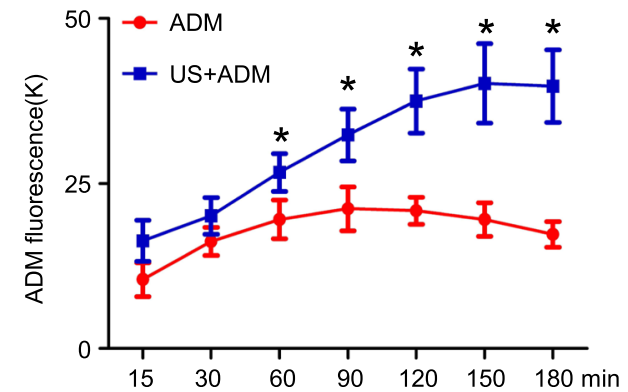

$30 \mathrm{~min}$

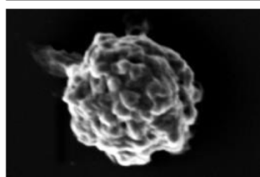

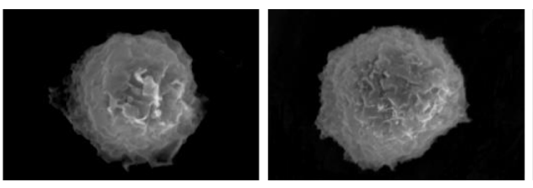
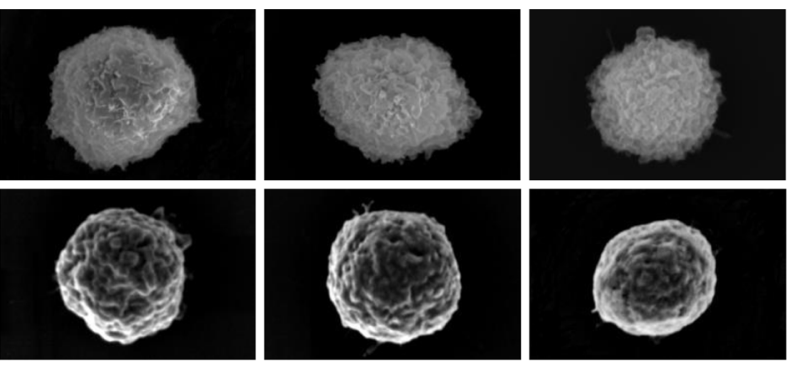

HEPG2/ADM
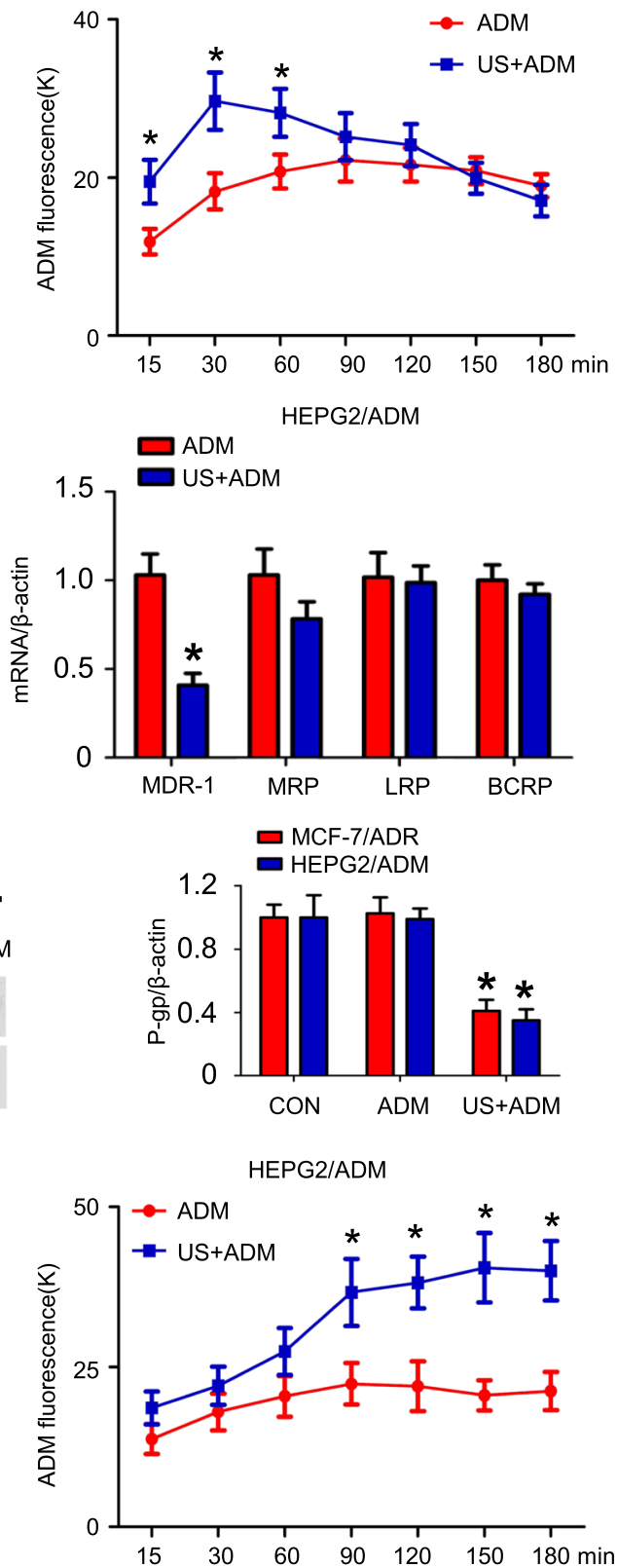

Fig. 2 (See legend on next page.) 
(See figure on previous page.)

Fig. 2 The mechanisms of US-mediated ADM accumulation. a Cell morphology and cytomembrane changes after US exposure $($ scale bar $=5 \mu m) ;(\mathbf{b})$ The dynamic change of ADM concentration in MCF-7/ADR and HEPG2/ADM cells treated with ADM immediately after US exposure; $N=3$; data are represented as mean \pm s.d; *P $<0.05$; (c) Q-PCR of drug efflux transporters mRNA in MCF-7/ADR and HEPG2/ADM cells in $24 \mathrm{~h}$ after US exposure or not; $\mathrm{N}=3$; data are represented as mean $\pm \mathrm{s.d}$; ${ }^{*} P<0.05$; (d) Immunoblotting of P-gp expression in $24 \mathrm{~h}$ after US exposure; $\mathrm{N}=3$; data are represented as mean \pm s.d; ${ }^{*} P<0.05$; (e) The dynamic change of ADM concentration in MCF-7/ADR and HEPG2/ADM cells treated with US+ADM or ADM alone. ADM was added to the mediums in $24 \mathrm{~h}$ after exposure to $\mathrm{US} ; \mathrm{N}=3$; data are represented as mean \pm s.d; ${ }^{*} P<0.05$

different response to ADM treatment might result from higher mitotic rate of cancer cells. The half-inhibitory concentration (IC50) dosage of ADM for MCF-ADR cells was used in the following experiments in vitro. Next, MCF-7/ADR cells and HUVEC cells were incubated with ADM and treated with ultrasound of different acoustic intensities. We assessed ADM accumulation and retention following US exposure. Expectedly, intracellular ADM concentrations were significantly enhanced in MCF-7/ADR cells with the increase in acoustic intensity $\left(P<0.05, \geq 0.40 \mathrm{~W} / \mathrm{cm}^{2}\right.$; Fig. 1c). Moreover, ultrasound acoustic intensity of $0.74,1.22 \mathrm{~W} /$ $\mathrm{cm}^{2}$ increased the quantity of ADM nuclei localization in MCF-7/ADR cells (Fig. 1e). We further explored whether US exposure could elevate the cytotoxicity of ADM against ADM-resistant cells. The viability of MCF-7/ADR cells with US+ADM treatment decreased significantly with the increase in ultrasound acoustic intensity $\left(P<0.05, \geq 0.74 \mathrm{~W} / \mathrm{cm}^{2}\right.$, Fig. $\left.1 \mathrm{~d}\right)$. In contrast, intracellular ADM concentrations in HUVEC did not increased after US exposure (Fig. 1c). The cell viability in HUVEC was slightly reduced after treated with $\mathrm{ADM}+1.22 \mathrm{~W} / \mathrm{cm}^{2}$ US exposure $\left(P<0.05\right.$, vs. $0 \mathrm{~W} / \mathrm{cm}^{2}$; Fig. 1d), which might result from US itself cytotoxicity. Considering $0.74 \mathrm{~W} / \mathrm{cm}^{2}$ US largely enhanced sensitivity to ADM of ADM-resistant cells but had minor effect on HUVEC, $0.74 \mathrm{~W} / \mathrm{cm}^{2}$ was selected as the optimal acoustic intensity in vitro.

In order to evaluate whether US exposure reversed MDR in other drug resistant cells, HEPG2/ADM cell line, another MDR cell line, was also utilized in the following experiments. The IC50 dosage of ADM for HEPG2/ADM cells was $10.26 \pm 1.29 \mu \mathrm{g} / \mathrm{ml}$. The IC50 dosage of ADM was used in the following studies involving HEPG2/ADM cells (Additional file 3: Figure S2B and Additional file 4: Table S2). Ultrasound acoustic intensity of $0.74 \mathrm{~W} / \mathrm{cm}^{2}$ increased the quantity of ADM nuclei localization in HEPG2/ADM cells (Fig. 1f). Furthermore, the viability of HEPG2/ADM cells treated with US+ADM was significantly lower than that of cell treated with ADM alone $(29.15 \pm 2.08 \%$ vs. $53.94 \pm 3.16 \% ; P<0.05$, Fig. $1 \mathrm{~g})$. The IC50 of ADM concentration in US+ADM treatment decreased $40 \%$ and $38 \%$ compared with $\mathrm{ADM}$ group in MCF-7/ADR and HEPG2/ADM cells, respectively $(P<0.05$; Additional file 4: Table S2 and Additional file 3: Figure S2A and B).
The decreased cell viability in US+ADM group is likely attributed to increased sensitivity to ADM-induced proliferation inhibition and apoptosis. We detected the proliferation profile of MCF-7/ADR and HEPG2/ADM cells using EDU staining. As shown in Fig. 1h, the treatment of US+ADM showed a decreased proliferative cell population in MCF-7/ADR and HEPG2/ADM cells, compared to ADM treatment $(42.81 \pm 4.83 \%, 39.76 \pm 4.07 \%$ vs $65.70 \pm 4.36 \%, 67.49 \pm 4.69 \% ; P<0.05$; respectively). Additionally, TUNEL assays and flow cytometry assays were performed to determine the apoptotic index of these cells. For TUNEL staining, the percentage of MCF-7/ADR and HEPG2/ADM cells with apoptotic features induced by ADM treatment was $37.6 \pm 4.92 \%$ and $39.45 \pm 7.60 \%$, respectively. When combined with US exposure, it increased to $73.19 \pm 9.82 \%$ and $75.72 \pm 9.01 \%$, respectively $(P<0.01$, compared with ADM group, Fig. 1i). Similar to these results, flow cytometry assays showed that apoptotic cell ratio at G2+G4 stage of MCF-7/ADR and HEPG2/ADM cells treated with $\mathrm{ADM}$ alone was $22.70 \pm 4.92 \%$ and $24.7 \pm 6.08 \%$ of cells, whereas $80.91 \pm 7.41 \%$ and $86.10 \pm 6.47 \%$ of cells treated with US+ADM, respectively $(P<0.01$, compared with ADM group, Additional file 3: Figure S2C).

\section{Sonoporation effect of US exposure briefly enhances the accumulation and retention of ADM in MDR cells}

We studied the potential mechanisms by which US exposure reversing MDR. We firstly explored whether ADM accumulation in response to US exposure is mediated by sonoporation effect which often disrupts the cell membrane. As is shown in Fig. 2a, cell membranes became crimpled and micropores development was observed immediately after exposure to US. However, the micropores disappeared and cytomembranes recovered normal shape at $24 \mathrm{~h}$ post-exposure. To investigate the role of US-induced sonoporation in reversal of MDR, we added ADM to culture dishes at $5 \mathrm{~min}$ after exposure to either US exposure or control treatment and monitoring intracellular ADM concentration. ADM concentrations peaked in 30 min post-treatment in US+ADM group and 90 min post-treatment in ADM group (Fig. 2b). The peak of ADM concentrations in US+ADM group were higher than that of ADM groups in MCF-7/ADR and HEPG2/ADM cells by more than $50 \%(P<0.05$; respectively). In $120 \mathrm{~min}$ post-treatment in US+ADM group, 
A
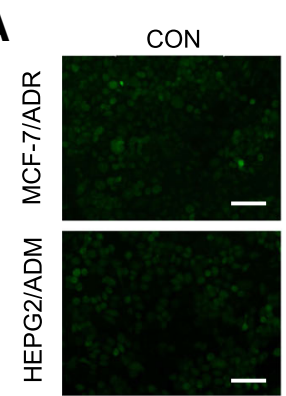

B

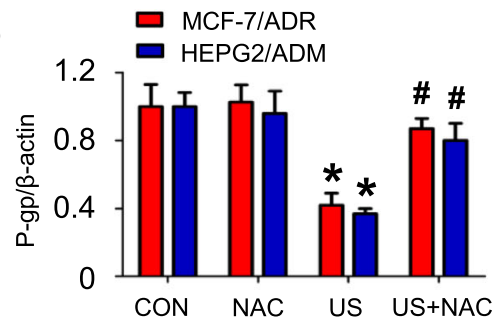

D

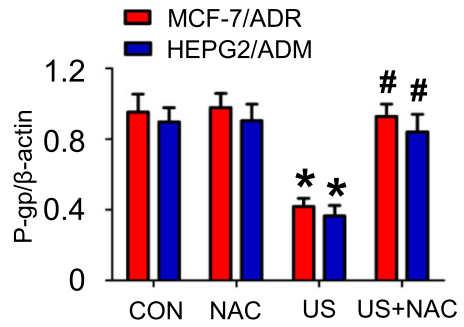

G

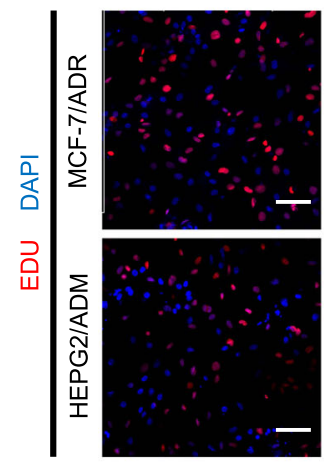

I

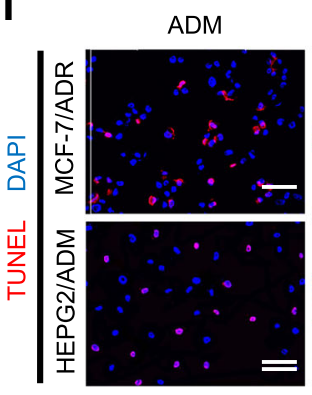

$\mathrm{NAC}+\mathrm{ADM}$

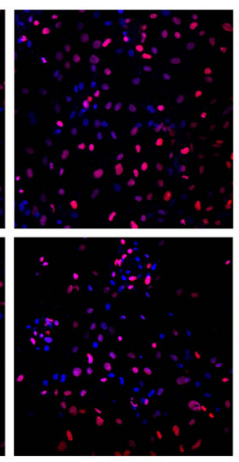

$\mathrm{NAC}+\mathrm{ADM}$

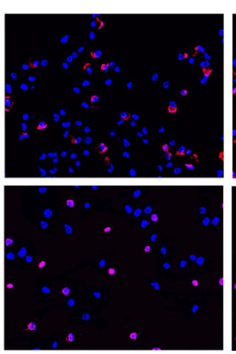

E

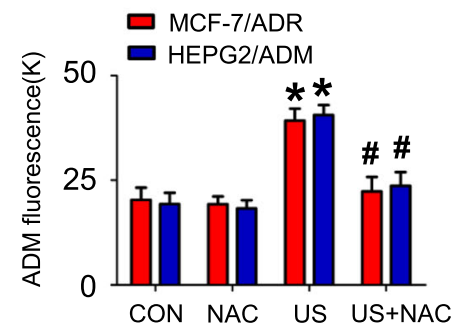

US+ADM
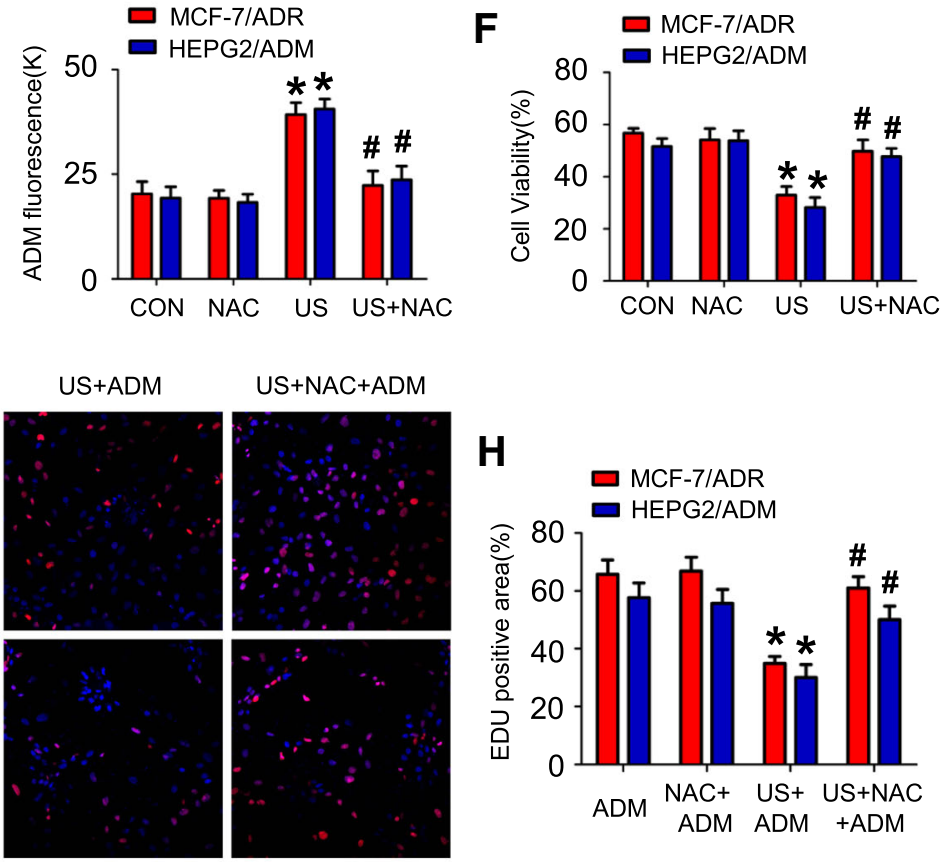

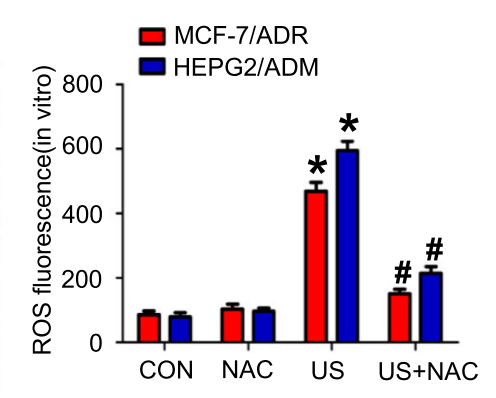

C
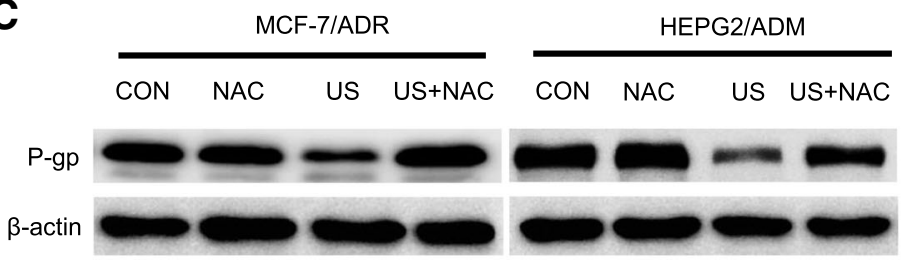

$\beta$-actin

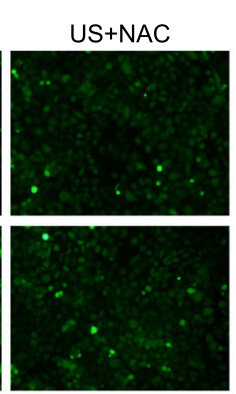


(See figure on previous page.)

Fig. 3 US exposure induces P-gp down-regulation is dependent on intracellular ROS generation. a US induced ROS generation in MCF-7/ADR and HEPG2/ADM cells which could be blocked by pre-treated with $5 \mathrm{mM} \mathrm{NAC}$ for $2 \mathrm{~h}$ (scale bar $=50 \mu \mathrm{m}$ ); Representative images of DCFH-DA staining in MCF-7/ADR and HEPG2/ADM cells $24 \mathrm{~h}$ post-treatment; $\mathrm{N}=3$; data are represented as mean \pm s.d; * $P<0.05$ compared with CON group; (b-d) Pre-treated with 5 mM NAC for 2 h inhibited US-induced P-gp down-regulation; Q-PCR assays (b), Western blot assays (c-d); (e-j) MCF-7/ADR and HEPG2/ADM cells were pre-treated with or without $5 \mathrm{mM} \mathrm{NAC}$ for $2 \mathrm{~h}$ before exposure to US+ADM. ADM intracellular concentration was determined by measuring fluorescent intensity (e); Cell viability was determined by MTT assay (f); Percentage of proliferative cell was determined by EDU staining $(\mathbf{g}-\mathbf{h}$, scale bar $=50 \mu \mathrm{m})$; Percentage of cell apoptosis was determined by TUNEL staining $(\mathbf{i}-\mathbf{j}$, scale bar $=50 \mu \mathrm{m}) ; N=3 ;$ data are represented as mean \pm s.d; ${ }^{*} P<0.05$ compared with ADM group

ADM concentrations decreased to the comparable levels as the ADM group in both cell lines $(P>0.05$; Fig. $2 b)$. These findings indicated sonoporation might mediate short-term effect of US exposure in enhancing ADM accumulation.

\section{Down-regulation of P-gp is mainly attributed to US-mediated MDR reversal}

US exposure has been reported to decrease drug transporter expression. We, therefore, explored whether US exposure reversed MDR through repressing drug efflux transporter expression. We detected the expression level of MDR-1, MRP, LRP and BCRP gene $24 \mathrm{~h}$ after treatment. P-gp (MDR-1) mRNA expression level in cells treated by US+ADM decreased more obviously than others (Fig. 2c). In agreement with these results, immunoblotting showed that both $\mathrm{P}$-gp protein expression was significantly decreased in $24 \mathrm{~h}$ after US exposure in MCF-7/ADR and HEPG2/ADM cells $(P<0.05$; respectively; Fig. 2d). To determine the role of P-gp down-regulation in US-mediated MDR reversal, we added ADM to culture dishes $24 \mathrm{~h}$ after cells were stimulated by US or control treatment. In US+ADM group, ADM concentrations showed a time-dependent increase and reached a plateau at $120 \mathrm{~min}$ post treatment in MCF-7/ADR cells and at $90 \mathrm{~min}$ in HEPG2/ADM cells (Fig. 2e). The peak of ADM concentrations in both US $+\mathrm{ADM}$ group were more than twice as much as that of ADM group in MCF-7/ADR and HEPG2/ADM cells ( $P$ $<0.05$; respectively). Taken together, these findings demonstrated a critical role of P-gp down-regulation in US-induced MDR reversal.

\section{ROS activation is responsible for US exposure mediated P-gp down-regulation}

Previous studies documented that US exposure modulated gene expression via the generation of ROS. We hypothesized US exposure reversed MDR via ROS mediated transcriptional repression of P-gp. As is shown in Additional file 5: Figure S3, US exposure significantly increased ROS activity in MCF-7/ADR and HEPG2/ADM cells with the time increase ( $\geq 4 \mathrm{~h}, P<0.05$, respectively). To determine if elevated ROS contributed to P-gp down-regulation, a ROS scavenger $\mathrm{N}$-acetyl-L-cysteine
(NAC) was used. It was showed that pre-treated with $5 \mathrm{mM}$ NAC could prevent US-mediated ROS activation $(P>0.05$; Fig. 3a). Moreover, Q-RTPCR assays and western blot assays showed that US-induced P-gp repression could be attenuated by NAC pre-treatment (US+NAC vs. US, $P<0.05$; Fig. $3 \mathrm{~b}, \mathrm{c}$ and $\mathrm{d}$, respectively). In addition, pre-treated with $5 \mathrm{mM}$ NAC significantly decreased intracellular ADM concentration (US+NAC + ADM vs. US + ADM, $P<0.05$; Fig. 3e), apoptotic cell ratio (US+NAC + ADM vs. US+ADM, $P<0.05$; Fig. $3 \mathrm{i}$ and $\mathrm{j})$ and increased cell viability and proliferative cell ratio (US+NAC + ADM vs. US+ADM, $P<0.05$, respectively; Fig. $3 f$, $g$ and $h)$ in MCF-7/ADR and HEPG2/ADM cells receiving US+ADM treatment.

\section{US exposure increases miR-200c/34a expression via oxidative stress}

ROS often fulfills its biological effect by modulating microRNAs (miRs) expression in cancer [28]. Thus, we hypothesized that oxidative stress-induced miRs serve as a link between US, ROS and P-gp downregulation. We detected the expression level of several oxidative stress-induced miRs that also regulate drug resistance in cancer on $24 \mathrm{~h}$ after US exposure. It was shown that miR-200c-3p and miR-34a-3p were markedly overexpressed in both cell lines after US exposure $(P<0.01$, respectively; Fig. 4a). The effect of US on miR-200c/34a expression could be abrogated by pretreatment with NAC (US+NAC vs. US, $P<0.05$; Fig. $4 \mathrm{~b}$ and $\mathrm{c}$ ). $\mathrm{H}_{2} \mathrm{O}_{2}$-induced miR-200c/34a upregulation further confirmed US exposure elevates miR-200c/34a expression depending on oxidative stress pathway $(P<0.05$; Fig. $4 d)$. Successively, we assessed miR-200c/34a effect on multidrug resistance. MCF-7/ADR cells was transfected with miR-200c/34a mimics or inhibitors. MiR-200c was shown to repress $\mathrm{P}$-gp-mediated MDR by targeting JNK-2/c-Jun pathway [29]. Consistent with previous studies, we observed that miR-200c significantly decreased P-gp expression in MCF-7/ADR cells (Fig. 4e). Using TargetScan programme, we found a potential miR-34a-3p binding sites on three prime untranslated region (3' UTR) of P-gp (ABCB1) (Fig. 4f). We further employed luciferase assay system to test whether miR-34a-3p directly binds to 3' UTR of P-gp. As is 
A

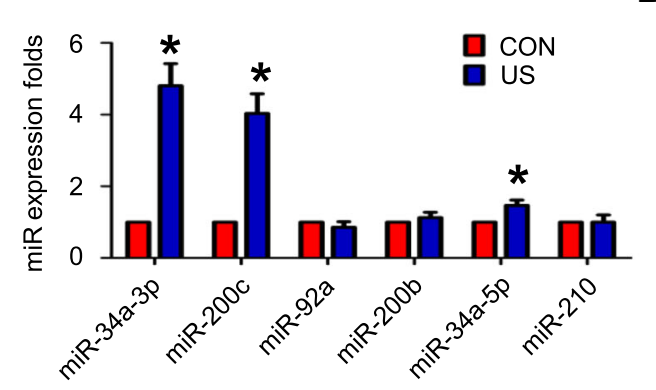

D

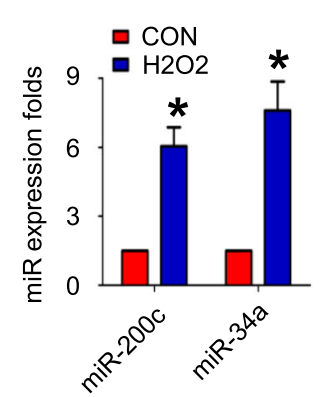

G
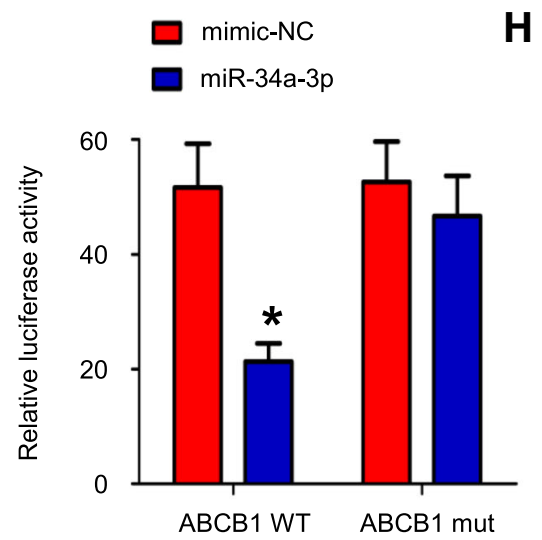

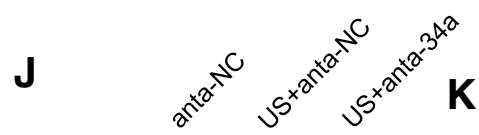
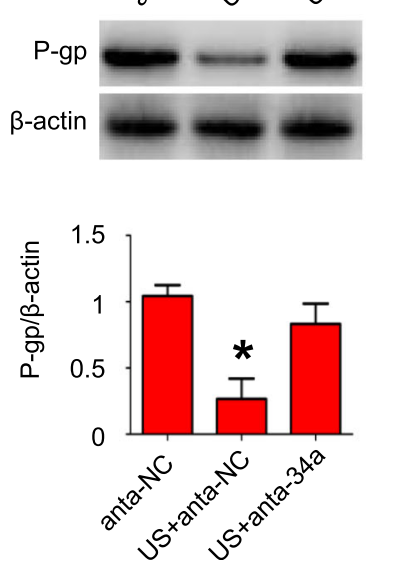

E
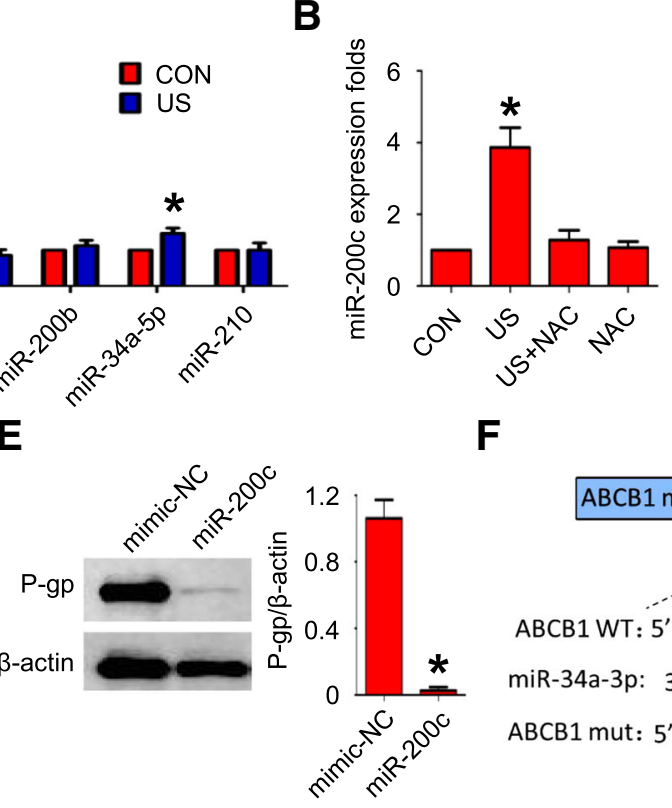

C

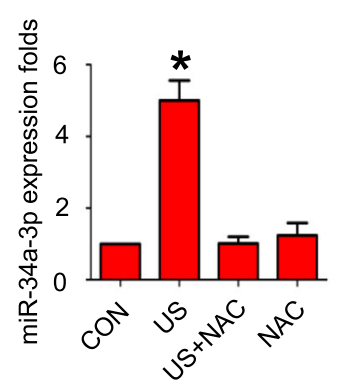

F

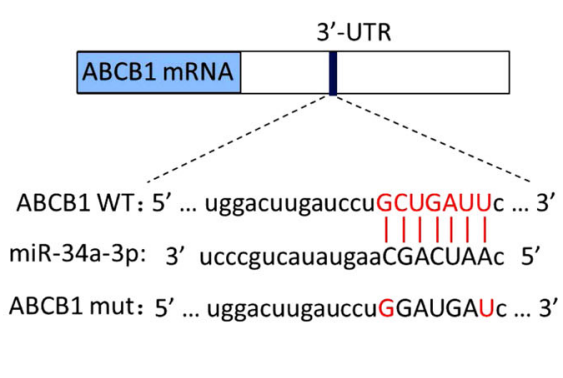

H
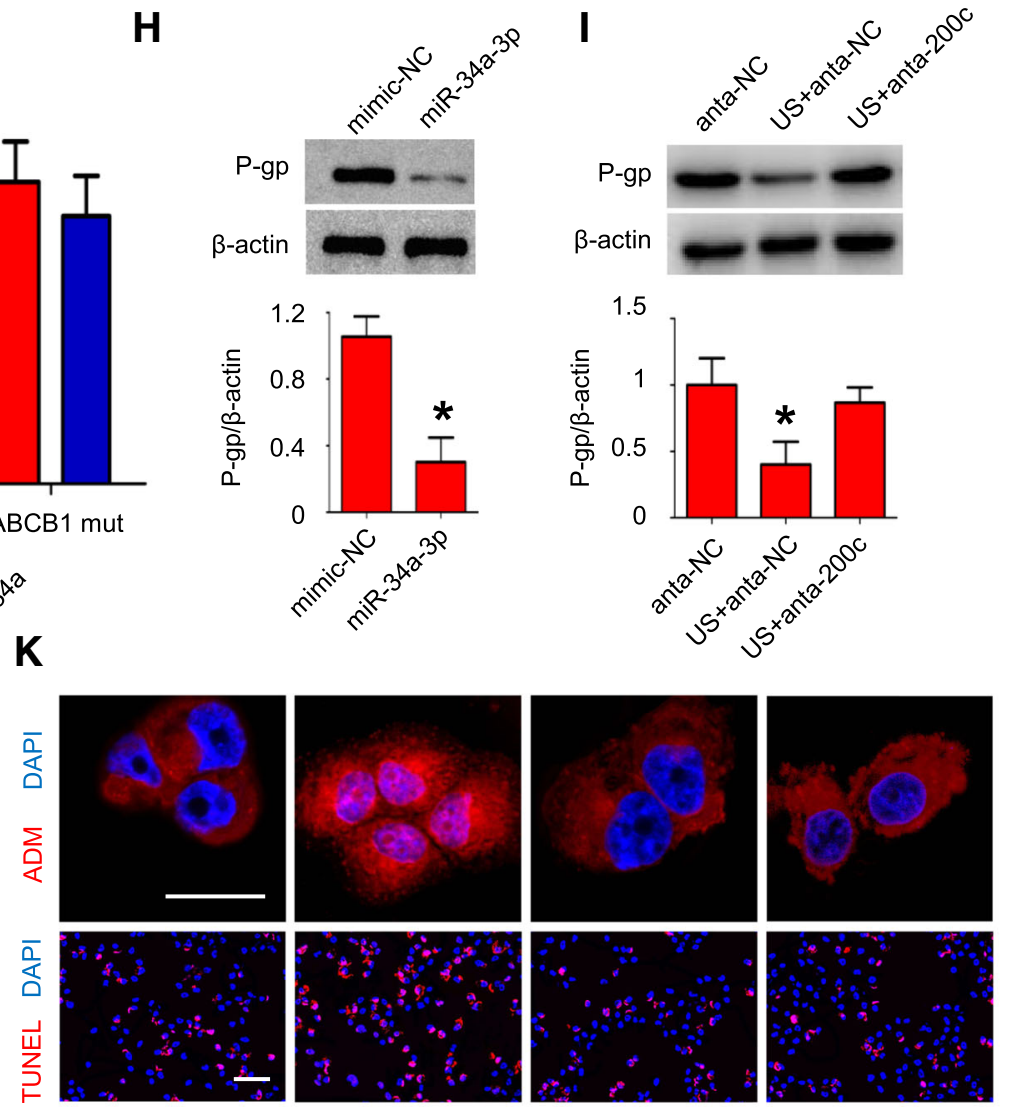

$\stackrel{\operatorname{ADM}}{+\operatorname{anta-NC}}$

US+ADM

US+ADM

+anta-200c

US+ADM

tanta-34a

Fig. 4 (See legend on next page.) 
(See figure on previous page.)

Fig. 4 miR-200c/34a participate in US decreasing P-gp expression. a Q-PCR of oxidative stress responsive miRNAs in MCF-7/ADR cells in $24 \mathrm{~h}$ after US exposure; $N=3$; data are represented as mean \pm s.d; $* P<0.05$; (b-c) Pre-treated with 5 mM NAC abrogated US-induced miR-200c (b) or miR-34a-3p (c) overexpression in MCF-7/ADR cells; $N=4$; data are represented as mean \pm s.d; ${ }^{*} P<0.05$ compared with CON group; (d) MiR-200c/34a expression in MCF-7/ADR cells treated with $400 \mathrm{mM} \mathrm{H}_{2} \mathrm{O}_{2} ; \mathrm{N}=3$; data are represented as mean \pm s.d; ${ }^{*} P<0.05$ compared with CON group; (e) $\mathrm{P}$-gp expression in MCF-7/ADR cells transfected with miR-200c mimic; $N=3$; data are represented as mean \pm s.d; ${ }^{*} P<0.05$; (f) Predicted miR-34a-3p seed sequence match to the sequence in the $3^{\prime}$ UTR of P-gp (ABCB1) mRNA; (g) Verification of ABCB1 as a target gene of miR-34a-3p by the dual luciferase reporter assay; ( $\mathbf{h}$ ) P-gp expression in MCF-7/ADR cells transfected with miR-200c mimic; $N=3$; data are represented as mean \pm s.d; ${ }^{*} P<0.05$; (i-j) $M i R-200 c$ (i) or miR-34a-3p (j) inhibition attenuated US-induced P-gp up-regulation in MCF-7/ADR cells; $N=3$; data are represented as mean \pm s.d; ${ }^{*} P<0.05$ compared with anta-NC group; (k) Images of intracellular ADM distribution (Upper, scale bar $=10 \mu \mathrm{m})$ and TUNEL staining (lower, scale bar $=50 \mu \mathrm{m})$ in MCF-7/ADR cells treated as described in (i-j); Quantitative analysis of ADM intracellular concentration and TUNEL-positive cell ratio was shown in Fig.S4A and S4B, respectively

shown in Fig. 4g, the luciferase activity was significantly reduced in miR-34a-3p overexpressing cells, whereas the mutant 3' UTR didn't reveal a significant response to miR-34a-3p. Expectedly, miR-34a-3p significantly decreased P-gp expression in MCF-7/ADR cells (Fig. 4h). In addition, miR-200c/34a inhibition attenuated US-induced P-gp down-regulation (Fig. 4i and j). The effect of US on enhancing ADM uptake and ADM-inducing apoptosis was diminished in the presence of miR-200c/34a inhibition (Fig. 4k, Additional file 6: Figure S4A and B). Furthermore, both miR-200c and miR-34a mimics enhanced ADM uptake, ADM-induced cytotoxicity, proliferation inhibition and apoptosis in MCF-7/ADR cells (Additional file 6: Figure S4C, D, E and F). These results indicated an important role of oxidative stress-induced miR-200c/34a in US-mediated P-gp downregulation.

MiR-200c/34a and ZEB1 formed a negative feedback loop Previous studies demonstrated the existence of ZEB1/ miR-200c double feedback loop is required for oxidative stress induced miR-200c [30]. Thus, we further investigated if US-induced oxidative stress triggers such double feedback loop. We revealed that US exposure decreased ZEB1 expression, which could be prevented by pretreatment of NAC (Fig. 5a). Moreover, chromatin immunoprecipitation followed next-generation sequencing (CHIP-Seq) in HEPG2 cells revealed that ZEB1 occupies regions at the promoters of miR-200c/34a host gene (Fig. 5b). QCHIP assay further confirmed the occupancy by endogenous ZEB1 at miR-200c/34a promoter in MCF-7/ADR cells (Fig. 5c). Accordingly, ZEB1 knockdown elevated miR-200c/34a expression level, indicating ZEB1 repressed miR-200c/34a transcription activity (Fig. 5d). We subsequently explore whether miR200c/34a targets ZEB1. Two potential miR-34a-3p binding sites on 3' UTR of ZEB1 were found by using TargetScan programme (Fig. 5e). Luciferase assay indicated both binding sites are functional (Fig. 5f). It is also known that miR-200c binds to ZEB1 3'UTR and decreases its expression level [31]. Expectedly, transfection of miR-200c mimic resulted in a significant reduction of luciferase activity of ZEB1 3'UTR in MCF-7/ADR cells (Fig. 5g).
Successively, we investigated whether miR-200c/34a affected ZEB1 expression. It turns out to be that miR-200c/ 34a overexpression reduced ZEB1 expression (Fig. $5 \mathrm{~h}$ and i). Moreover, knockdown of ZEB1 reduced P-gp expression. MiR-200c/34a inhibition could reverse the effect of ZEB1 knockdown on reducing P-gp expression (Fig. 5j). Taken together, miR-200c/34a and ZEB1 interplays and formed a double negative feedback loop. US triggered this circuitry by activating oxidative pathway, resulting in P-gp down-regulation finally.

\section{US exposure reverses MDR in vivo}

To evaluate whether US exposure reverses MDR in vivo, we established MCF-7/ADR xenograft models by subcutaneously inoculating nude mice with MCF-7/ADR cells. We first determined the optimal US Parameters in vivo. MCF-7/ADR xenograft from 24 tumor bearing mice was randomly subjected to ADM $(8 \mathrm{mg} / \mathrm{kg}$, i.v.) combined with US irradiation in six different acoustic intensities $\left(0,0.09,0.21,0.40,0.74\right.$, and $\left.1.22 \mathrm{~W} / \mathrm{cm}^{2}\right)$. We evaluated therapeutic sensitivity of each group by detecting ADM concentration, apoptotic and proliferative index in xenografts one week after treatment. ADM concentrations in MCF-7/ADR tumor tissue were significantly enhanced at $0.40 \mathrm{~W} / \mathrm{cm}^{2}, 0.74 \mathrm{~W} / \mathrm{cm}^{2}$, and $1.22 \mathrm{~W} / \mathrm{cm}^{2}$ acoustic intensity, compared with $0 \mathrm{~W} / \mathrm{cm}^{2}$ acoustic intensity $(P<0.05$, respectively, Fig. 6a). As for peritumoural muscle tissue, ADM concentration was not significantly enhanced with the increase in acoustic intensity $(P<0.05$, Fig. 6a). Furthermore, the percentage of apoptotic cells were significantly higher in MCF-7/ADR tumor tissue treated with ADM in combination with $0.40 \mathrm{~W} / \mathrm{cm}^{2}(15.41 \pm 3.60 \%), 0.74 \mathrm{~W} / \mathrm{cm}^{2}(28.93 \pm 3.77 \%)$, and $1.22 \mathrm{~W} / \mathrm{cm}^{2}(34.24 \pm 4.94 \%)$ US intensity, compared with $0 \mathrm{~W} / \mathrm{cm}^{2}$ US intensity $(10.48 \pm 1.49 \%$; $P<0.05$, respectively), whereas apoptotic cell ratio of peritumoural muscle tissue was significantly higher under $1.22 \mathrm{~W} / \mathrm{cm}^{2}$ US exposure than $0 \mathrm{~W} / \mathrm{cm}^{2}$ acoustic intensity $(8.98$ $\pm 1.46 \%$ vs. $5.41 \pm 0.74 \% ; P<0.05$; Fig. $6 \mathrm{~b}$ and c). To avoid the effect of US itself cytotoxicity, $0.74 \mathrm{~W} / \mathrm{cm}^{2}$ was also determined to be the optimal acoustic intensity in vivo and used in the following in vivo experiments. 
A
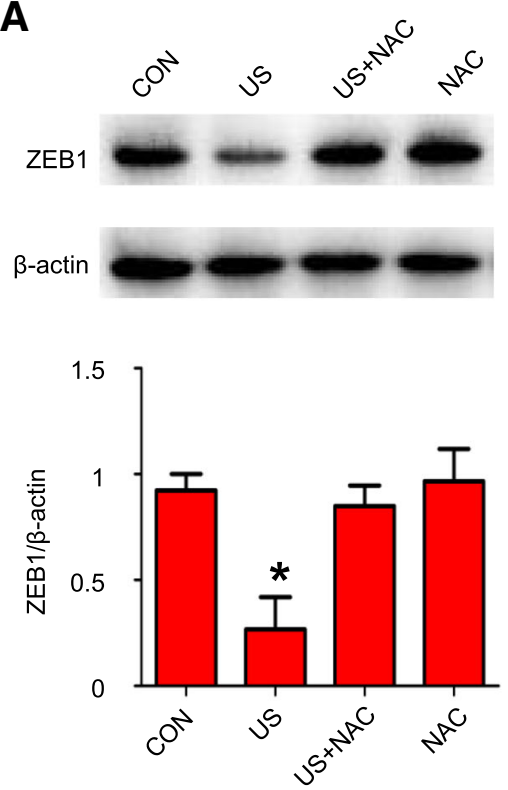

B

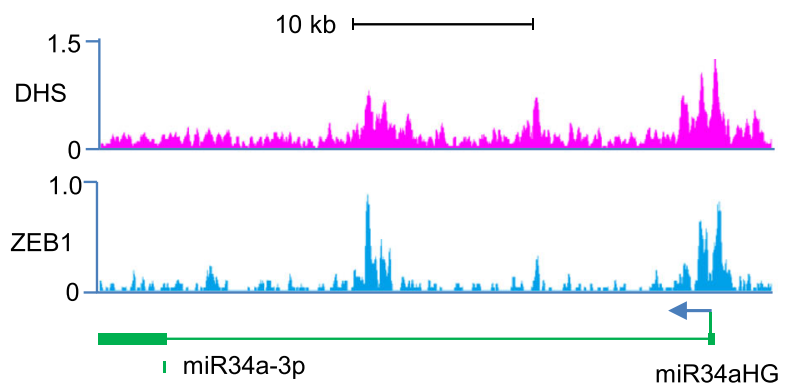

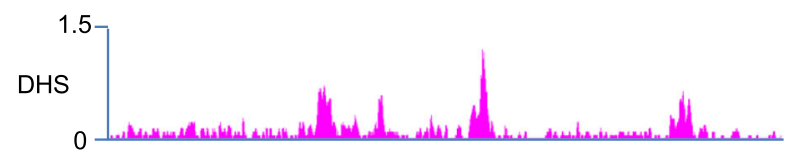

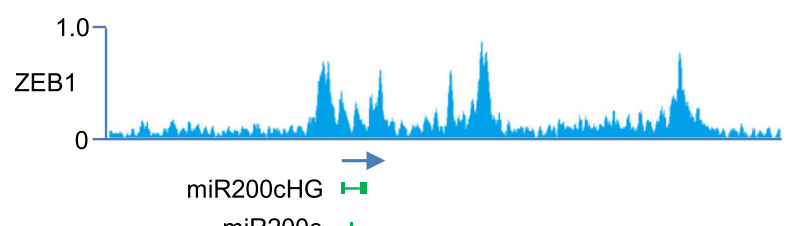

miR200c

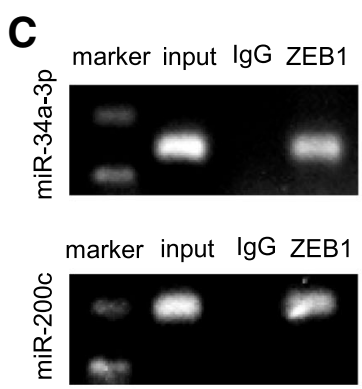

F

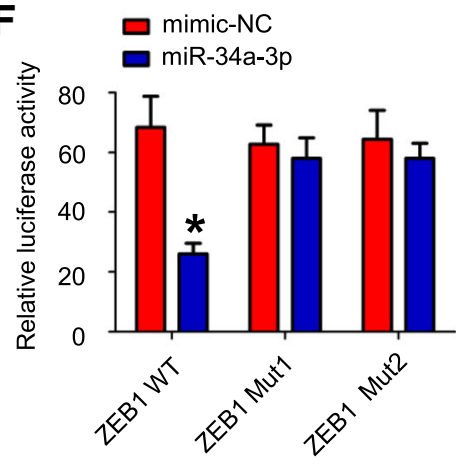

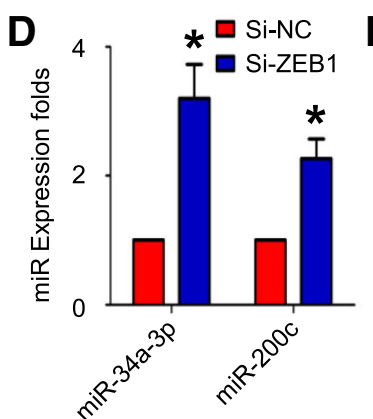

G

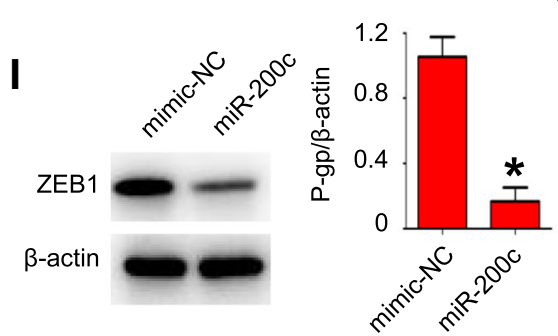

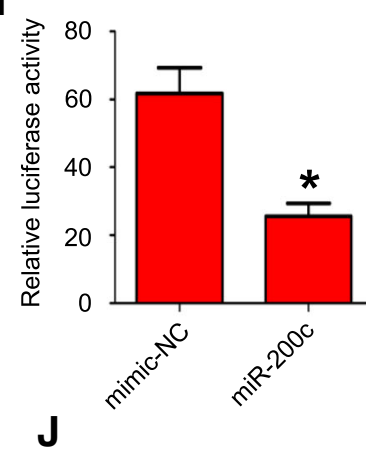

J

Si-NC

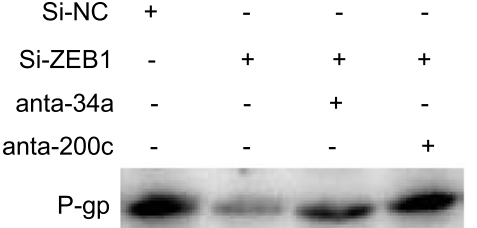

P-gp

$\beta$-actin
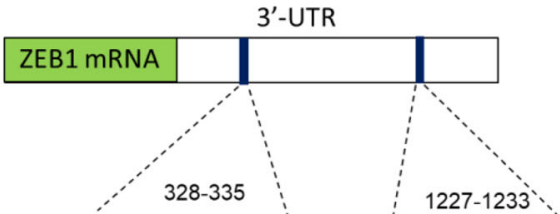

uagCUGAUUú....3

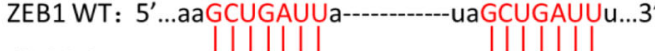

miR-34-3p: $3^{\prime}$...aaCGACUAAc...5' 3'...aaCGACUAAc...5' ZEB1 mut1: 5'...aaGCAUGAUa------------uaGCUGAUUu...3' ZEB1 mut2: 5'...aaGCUGAUUa---.---.----uaGCAUGAUu...3'

H
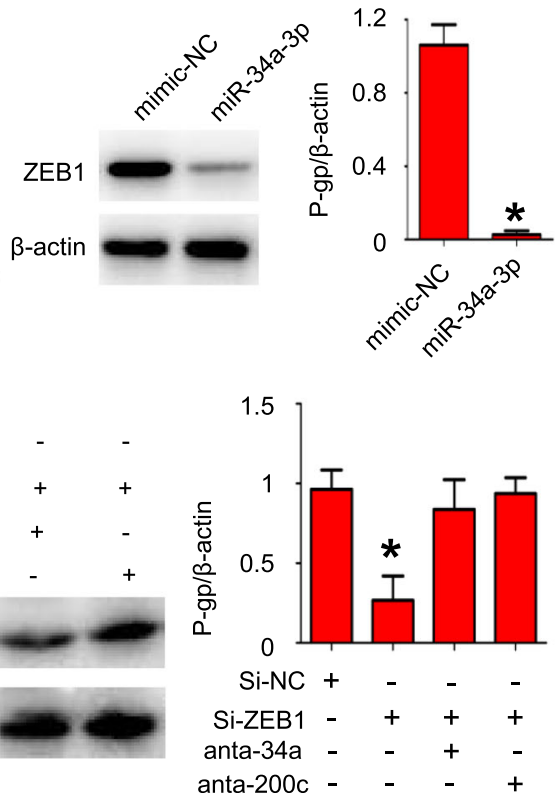

Fig. 5 (See legend on next page.) 


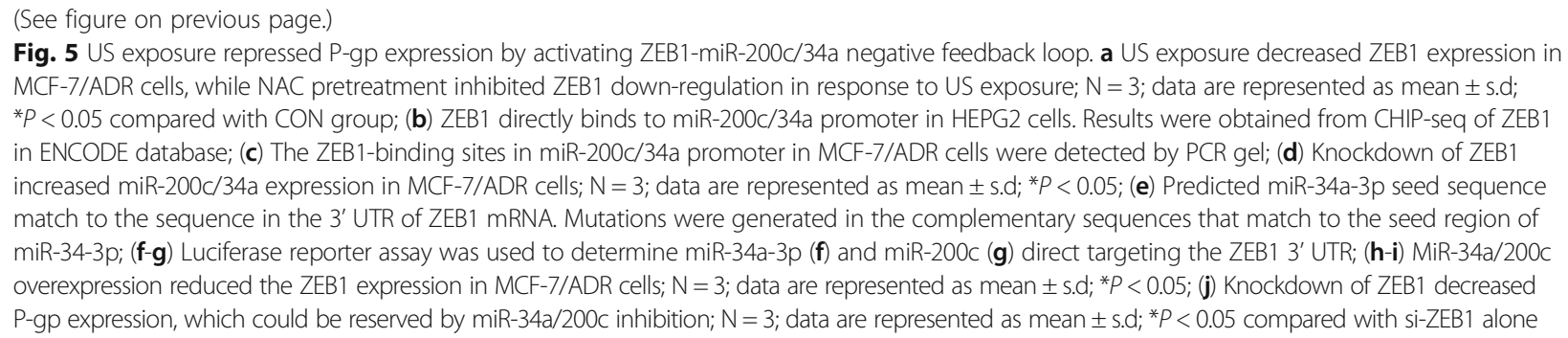

We investigated the longer-term beneficial effects of $0.74 \mathrm{~W} / \mathrm{cm}^{2}$ US exposure on reversal of MDR in vivo. MCF-7/ADR xenograft nude mice were randomly divided into ADM group and US+ADM group. Tumor growth and therapeutic sensitivity were monitored during the course of respective treatment. Xenograft tumor growth curves showed that tumors with ADM treatment alone continued to grow at a steady rate, whereas tumors in US+ADM group grew more slowly (Fig. 6f). Moreover, tumors showed significantly smaller increase of tumor size (Fig. 6d and e) and tumor weight (Fig. 6g) after US+ADM treatment compared with ADM treatment alone $(P<0.05$, ADM group vs. US+ADM group; respectively). These results indicated that US exposure and ADM treatment impairs MDR tumor growth synergistically in vivo. Moreover, we detected ADM concentration, proliferative and apoptotic index in each group at day 24. ADM concentration in MCF-7/ADR xenograft tissue treated with US+ADM is significantly higher than that in xenograft tissue treated with ADM alone $(P<0.05$, Fig. 6h). To investigate whether US exposure promotes ADM-mediated apoptosis in MDR tumors, TUNEL staining was used to detected apoptotic tumor cells after 24 days of treatment. As is shown in Fig. 6i, apoptotic tumor cells ratio was significantly higher in US $+\mathrm{ADM}$ groups $(65.12 \pm 7.08 \%)$ than $\mathrm{ADM}$ groups $(12.72$ $\pm 1.09 \% ; P<0.05$, respectively). The percentage of ki67 positive cell in US+ADM groups was significantly lower than ADM group $(P<0.05$, Fig. 6j). Taken together, these in vitro and in vivo experiments demonstrated that US exposure could overcome cancer MDR.

\section{US exposure decreases P-gp expression via ROS-ZEB1- $\mathrm{miR200c/34a} \mathrm{pathway} \mathrm{in} \mathrm{vivo}$}

To further confirm the relationship between US exposure, ROS, ZEB1, miR-200c/34a and P-gp, we analyzed their expression in isolated tumors from MCF-7/ADR xenograft nude mice. Consistent with the results in vitro, ROS activity in the US+ADM group was significantly higher than that in the ADM group $(P<0.05$; Fig. 7a). Furthermore, qRT-PCR assays indicated miR-200c/34a expression level were significantly higher in the US+ADM group than ADM group $(P<0.05$; Fig. $7 \mathrm{~b})$. Immunohistochemistry and western blotting showed that the expression of ZEB1 was significantly lower in US+ADM group, compared to ADM group ( $P<0.05$; Fig. $7 \mathrm{c}$ and d; respectively). Moreover, P-gp expression was significantly lower in US+ADM group than that in ADM group $(P<0.05$; Fig. $7 \mathrm{e}, \mathrm{f}$ and g; respectively).

\section{The effect of US exposure on drug-sensitive t cells}

We also explored the effect of US exposure on drug-sensitive cancer cells using MCF-7 and HEPG2 cell lines. Similar to our observations on MCF-7/ADM and HUVEC cells, cell viability was not significantly affected in neither MCF-7 cells nor HEPG2 cells using ultrasound acoustic intensity $\leq 0.74 \mathrm{~W} / \mathrm{cm}^{2}$ exposure $(P<0.05, \geq 0.74 \mathrm{~W} / \mathrm{cm} 2$, respectively; Additional file 7 : Figure S5C). The IC50 dosage of ADM for MCF-7 and HEPG2 cells was $1.82 \pm 0.34$ and $1.68 \pm 0.23 \mu \mathrm{g} / \mathrm{ml}$, respectively. The IC50 dosage of ADM and $0.74 \mathrm{~W} / \mathrm{cm}^{2} \mathrm{US}$ was used in the relevant experiments. There was almost no difference in IC50 of ADM between ADM + US treatment groups and ADM treatment groups $(P>0.05$, respectively; Additional file 7: Figure S5A, B and Additional file 4: Table S2). Confocal microscopy images revealed US exposure has no effect on the quantity of ADM nuclei localization in MCF-7 or HEPG2 cells (Additional file 7: Figure S5D). Furthermore, ADM concentration in MCF-7 or HEPG2 cells was not significantly changed in $24 \mathrm{~h}$ after US exposure $(P>0.05$, respectively; Additional file 7: Figure S5E), which is similar to our observations in HUVEC. Moreover, we investigated whether US induces P-gp down-regulation on these non-drug resistant cells by promoting ROS generation. The western blot analysis revealed that the $\mathrm{P}$-gp expression levels were remarkably lowered in MCF-7, HEPG2 and HUVEC cells compared with the MDR variants (Additional file 8: Figure S6B and C). Expectedly, US exposure significantly increased ROS activity in MCF-7, HEPG2 and HUVEC cells $(P<0.05$, respectively; Additional file 8: Figure S6A). However, we did not detect a significant reduction on P-gp expression of these cells $24 \mathrm{~h}$ after US exposure $(P>0.05$, respectively; Additional file 8: Figure S6B and C). Similarly, when we added ADM to culture dishes in $24 \mathrm{~h}$ after cells were exposed to US or control treatment, ADM intracellular concentration was not significantly increased in US groups, compared to control groups $(P>0.05$, respectively; 
A

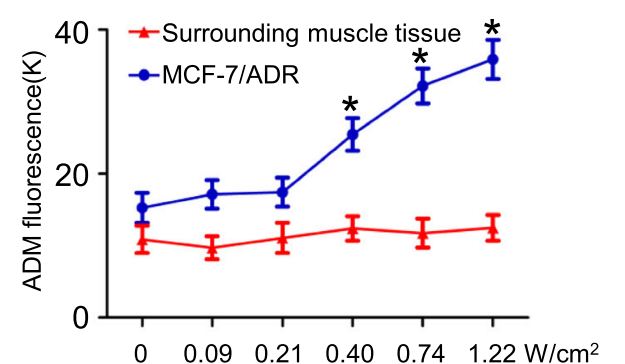

C
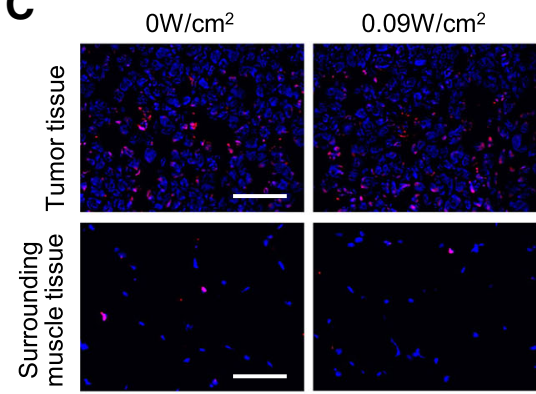

D

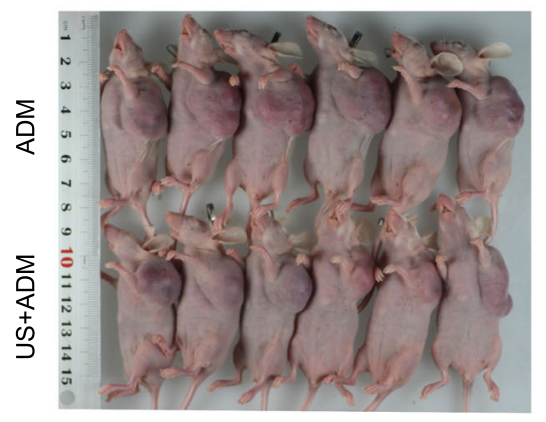

$\mathbf{F}$

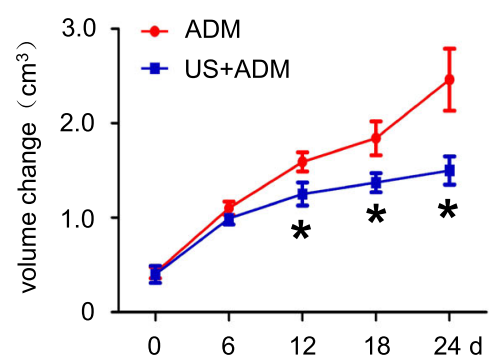

I
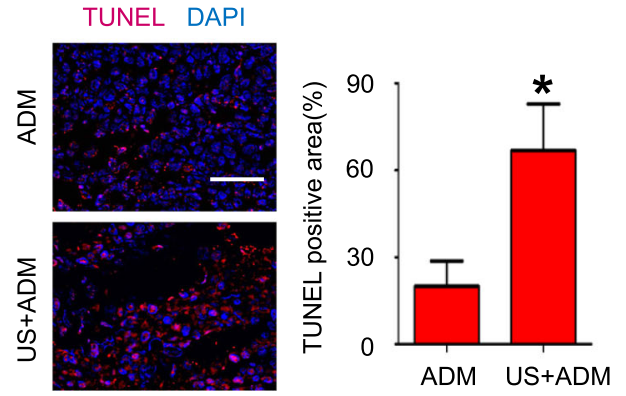

E

G
B

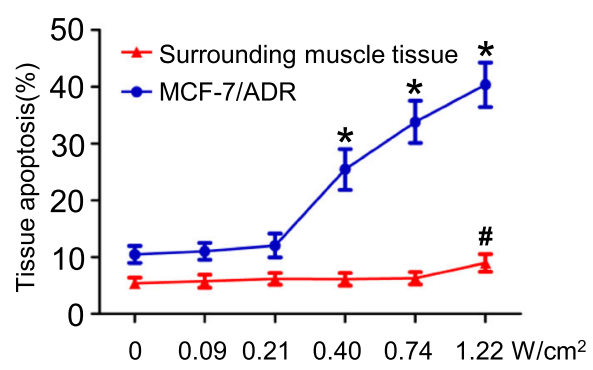

$0.21 \mathrm{~W} / \mathrm{cm}^{2}$

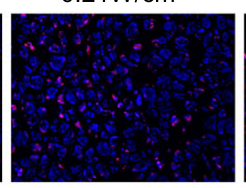

$0.40 \mathrm{~W} / \mathrm{cm}^{2}$

$0.74 \mathrm{~W} / \mathrm{cm}^{2}$

$1.22 \mathrm{~W} / \mathrm{cm}^{2}$
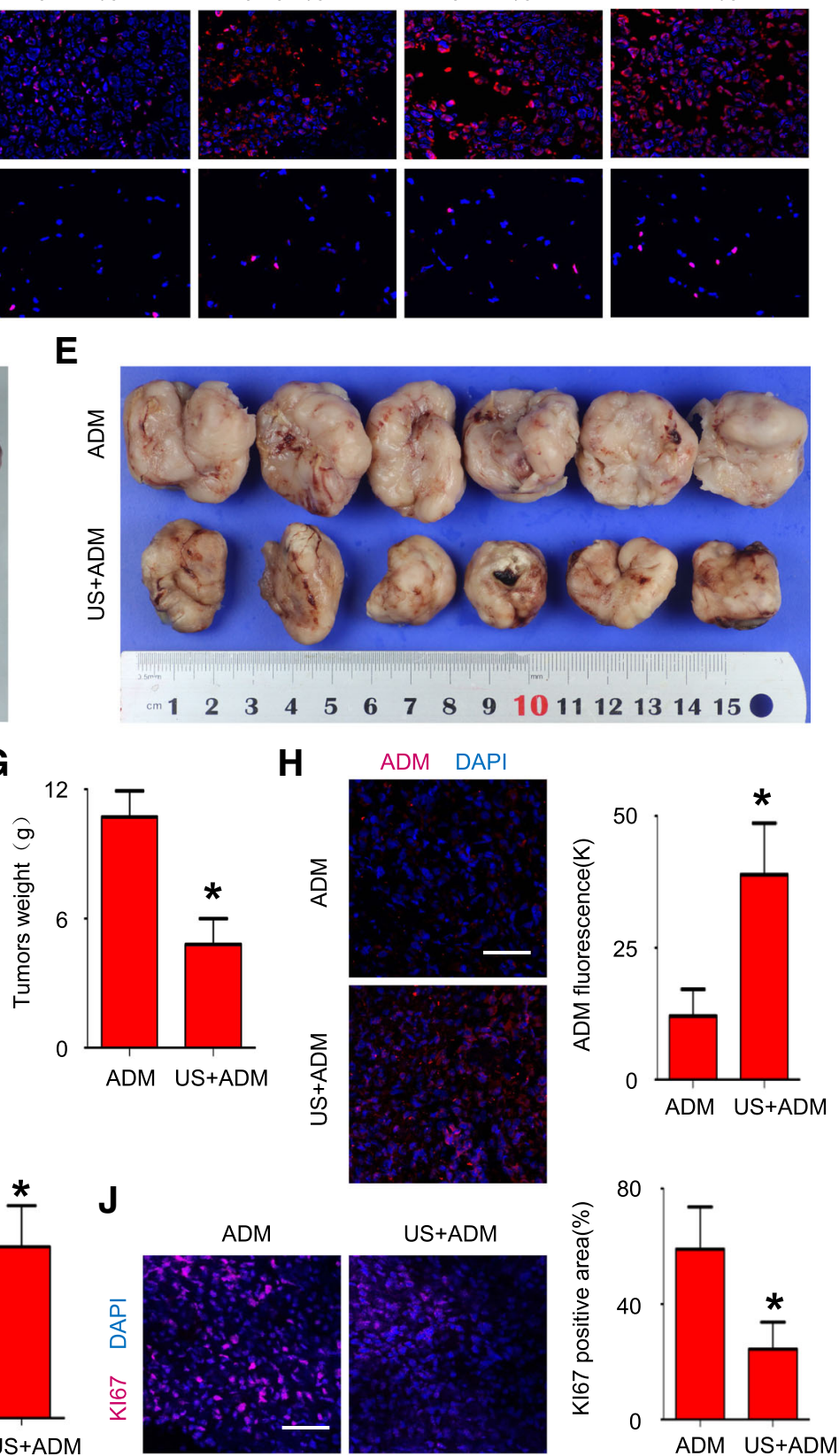

Fig. 6 (See legend on next page.) 


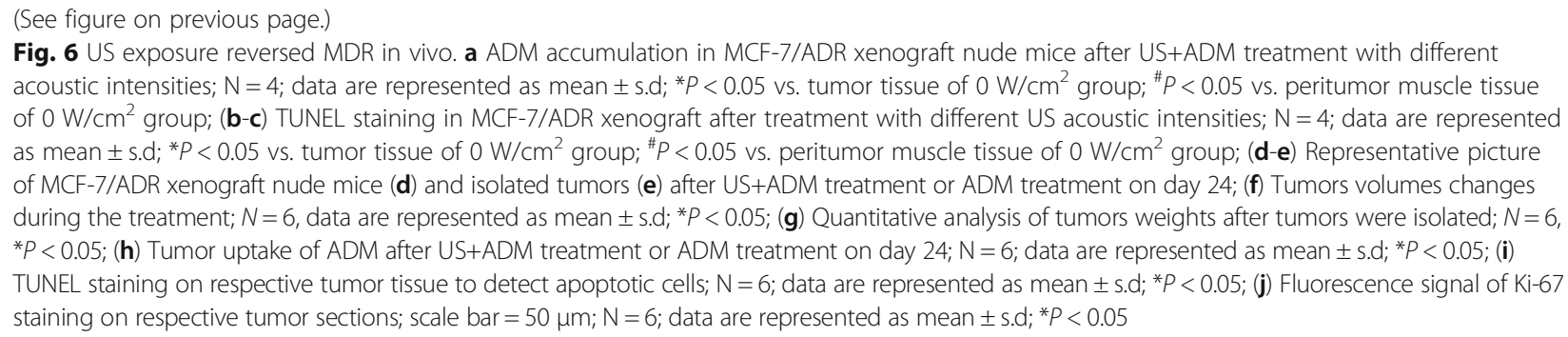

Additional file 8: Figure S6E). We found that sonoporation effect of US induced transient membrane perforation on MCF-7, HEPG2 and HUVEC cells, which almost disappeared in $60 \mathrm{~min}$ after US exposure (Additional file 8: Figure S6D). When we added ADM to culture dishes at 5 min after US exposure, ADM was transiently and mildly accelerated into MCF-7, HEPG2 and HUVEC cells (Additional file 8: Figure S6F). However, in $60 \mathrm{~min}$ post-treatment, ADM concentrations in US+ADM group were not significantly higher than that in ADM group $(P>0.05$, respectively; Additional file 8: Figure S6F). Collectively, our results suggested US exposure could not significantly affect cytotoxicity of ADM or P-gp expression for non-drug resistant cells.

\section{Discussion}

In this study, we found that $0.74 \mathrm{~W} / \mathrm{cm}^{2}$ US exposure increased ADM intracellular concentration and enhanced ADM cytotoxicity against MDR cancer cells both in vitro and in vivo. Compared with $\mathrm{ADM}$ treatment alone, combining ADM and US exposure reduced tumor growth rate and improved long-term prognosis in MCF-7/ ADR xenograft mice with no obvious increase in systemic toxicity. Mechanistically, US exposure promoted intracellular ROS generation and miR-34a/miR-200c expression. US-induced miR-34a/miR-200c and ZEB-1 formed a double-negative feedback loop to regulate P-gp expression and MDR phenotype.

In this study, we proposed US exposure as a novel treatment method for overcoming MDR without obvious side effects, and our results have confirmed this assumption. Our in vitro and in vivo data showed that $0.74 \mathrm{~W} /$ $\mathrm{cm}^{2}$ US exposure significantly increased intracellular ADM concentrations in ADM-resistant cells. In addition, we observed that a combination of ADM and US exposure resulted in a enhanced inhibition of apoptosis and proliferation in ADM-resistant cells compared with ADM treatment alone. More importantly, the combination of ADM and US exposure remarkably decreased tumor volume and improved prognosis in MCF-7/ADR xenograft mice. Our results are consistent with previous in vitro studies in which US exposure significantly increased the antitumor effect of ADM in neuroblastoma and ovarian MDR-variant cell lines [32,
33]. Particularly noteworthy, US exposure has several advantages over classical P-gp inhibitors. First, in contrast to chemical approach, US exposure reduced nonselective action on P-gp expressed in normal tissues by accurately targeting tumors, thus avoiding the systemic side-effects of classical P-gp inhibitors. This could be partly supported by the result in our experiments which showed that the combination of ADM and US exposure did not result in elevated deaths or obvious body weight loss amongst the tumor-bearing mice. This improvement is especially relevant for treating localized solid tumors. Moreover, because US treatment is a physical energy, the harmful interaction between P-gp inhibitors and other chemotherapy drugs can be avoided. All of these findings indicate that US exposure is a targeted, efficient, and safe treatment for cancer MDR.

The current study also demonstrated that increased ADM concentrations and reversal of MDR by US exposure was mainly due to decreased expression of P-gp expression. Previous studies have reported that US exposure temporarily increased intracellular drug retention in drug-sensitive cells [34]. In this study, we also observed that intracellular ADM concentrations of MDR cells increased mildly and temporarily when ADM administration was performed immediately after US exposure. Nonetheless, when ADM administration was performed $24 \mathrm{~h}$ after US exposure, substantially increased ADM concentrations could be stably maintained for more than $12 \mathrm{~h}$. Further study showed that the short-term effects of US exposure mainly can be ascribed to elevated cell membrane permeability caused by the sonoporation effect, whereas long-term effects resulted from transcriptional repression of P-gp expression. Compared with the sonoporation effect, down-regulation of P-gp yielded greater ADM accumulation over a longer duration. Therefore, it is reasonable to deduce that down-regulation of P-gp expression may be the main mechanism by which US exposure increased ADM accumulation in MDR cancer cells. Overexpression of the membrane drug efflux pump P-gp is one of the major mechanisms by which cancer cells develop MDR. The findings that US irradiation reduced P-gp expression further suggest that US irradiation may be a potential anti-MDR treatment. Interestingly, as a promising 


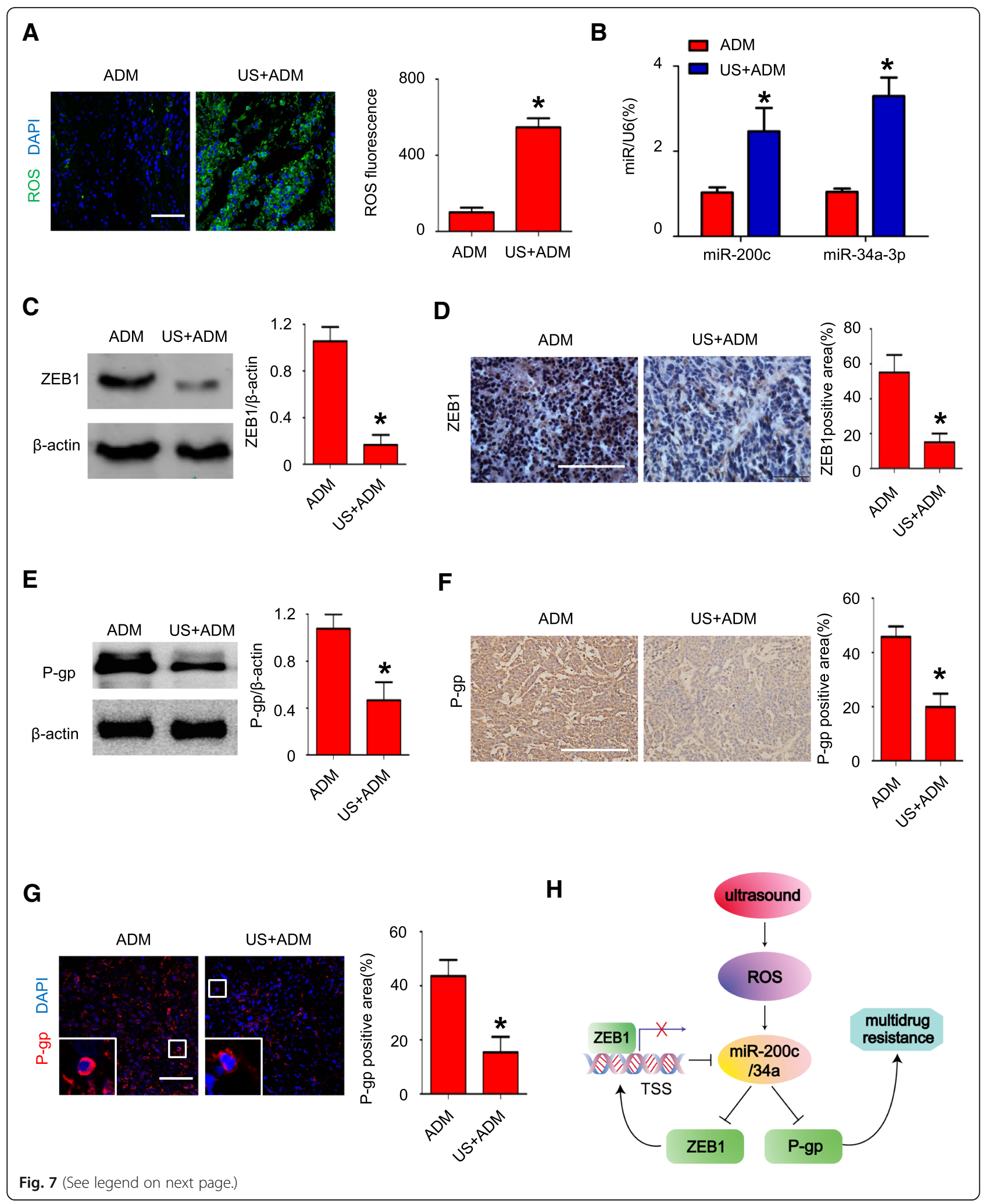




\section{(See figure on previous page.)}

Fig. 7 The effect of US exposure on ROS-ZEB1-miR200c/34a-P-gp pathway in vivo. a ROS staining (green) of tumor tissue in MCF-7/ADR xenograft nude mice treated with ADM alone or US+ADM; scale bar $=50 \mu \mathrm{m} ; \mathrm{N}=6$; data are represented as mean \pm s.d; ${ }^{*} P<0.05$; (b) MiR-200c/ 34a expression levels were quantified by qRT-PCR in MCF-7/ADR xenograft after US+ADM treatment or ADM treatment on day 24; $N=6$; data are represented as mean \pm s.d; ${ }^{*} P<0.05$; (c-d) Detecting ZEB1 expression in respective tumor tissue by western blot (c) and immunochemistry (d, scale bar $=10 \mu \mathrm{m}) ; \mathrm{N}=6$; data are represented as mean \pm s.d; ${ }^{*} P<0.05 ;(\mathbf{e}-\mathbf{g})$ Detecting P-gp expression in respective tumor tissue by western blot $(\mathbf{e})$, immunochemistry $(\mathbf{f}$, scale bar $=10 \mu \mathrm{m})$, and immunofluorescence $(\mathbf{g}$, scale bar $=100 \mu \mathrm{m}) ; \mathrm{N}=6$; data are represented as mean $\pm \mathrm{s}$.d; ${ }^{*} P$ $<0.05$; (h) Illustration of reversal of MDR mediated by US exposure in MDR cancer cells; Ultrasound exposure increases miR-200c/34a expression by promoting ROS generation. MiR-200c/34a overexpression directly or indirectly inhibits ZEB1 and P-gp expression. Down-regulation of ZEB1 in turn decreases its transcriptional repression on miR200c/34a. P-gp inhibition sensitizes MDR cells to MDR-associated drugs and increases the cytotoxicity of these chemotherapeutics

strategy, transcriptional repression is not only effective, but also enables the prevention of P-gp expression during the progression of disease [35]. It has been noted that in some tumors, P-gp expression is low before exposure to chemotherapy drugs, but increases after chemotherapy and eventually results in MDR [36]. Future studies should determine whether US irradiation started during the early stage of chemotherapy could prevent the occurrence of the MDR phenotype and improve the efficacy of treatment.

In this study, we revealed that the ability of US irradiation to repress P-gp expression might be based on the generation of ROS. It is known that US irradiation can promote ROS production as a consequence of the cavitation phenomena, which may result in ectopic expression of genes [37]. Moreover, previous studies also revealed evidence supporting the role of oxidative stress in down-regulating P-gp expression [38-41]. In accordance with previous studies [42], our immunofluorescence results showed that US exposure increased intracellular ROS production. More important, administration with NAC, a well-known ROS inhibitor, significantly blocked the US-mediated ROS generation, and almost abrogated US-induced P-gp inhibition. These findings suggest that decreased P-gp expression following US treatment might be mediated by elevated ROS.

MiR-200c and miR-34a could be induced by oxidative stress in several cell types, and are designated as oxidative stress-responsive miRNAs [30, 43, 44]. In this study, we found that US radiation increased mir-200c and miR-34a expression through oxidative signal pathway, which was responsible for P-gp down-regulation. Tumor suppressor miR-34a-5p is often down-regulated in drug-resistant cells $[45,46]$. Generated together with miR-34a-5p, miR-34a-3p has a similar expression level and functional role in different cells and tumor samples [47-49]. We found a remarkable increase in miR-34a-3p expression after US exposure, whereas a modest increase in miR-34a-5p. We further demonstrated miR-34a-3p could inhibit P-gp expression by directly binding to P-gp three prime untranslated region (3'-UTR). Repressing miR-34a-3p expression attenuated US-induced P-gp down-regulation, indicating the involvement of miR-34a-3p in US-mediated MDR reversal.
Additionally, miR-200c has been demonstrated to reverse P-gp-mediated MDR by blocking JNK2/c-Jun pathway [29, 50]. Consistent with previous studies, we also found overexpression of miR-200c reduced P-gp expression and combated MDR phenotype, while miR-200c inhibition reversed US-mediated P-gp down-regulation. These results indicate the involvement of miR-200c/34a in US-mediated MDR reversal, and that miR-200c/34a could be potential targets in treating drug-resistance.

It is known that oxidation stress alters miRNA expression by affecting their transcriptional activity [51]. The underlying mechanisms include well-characterized redox-responsive alterations in activity of transcriptional factors (TFs) [52]. ZEB1 is a well-described transcription repressor that is associated with mi-200c/34a down-regulation [53, 54]. Importantly, it has been discovered that oxidation stress could increase miR-200c expression by forming miR-200c/ZEB1 double negative feedback loop [30]. In keeping with previous studies, we found that US exposure triggered miR-200c/ZEB1 circuitry through oxidative pathway. Moreover, we also found that miR-34a and ZEB1 could also form a double negative feedback loop. The presence of mir-200c/34a/ZEB1 circuitry may convey robustness to anti-MDR effect of US and prevent the disturbance emanating from the cellular environment.

Furthermore, we found that US exposure had no effect on the cytotoxicity of ADM for MCF-7, HEPG2 and HUVEC cells. We further uncovered that US could increase ROS activity, but could not significantly decrease P-gp expression in these cells. Our results are similar to previous studies showing targeting P-gp was less effective in treating MCF-7 cells than that in treating MCF-7/ADR cells $[55,56]$. These probably result from the rather low baseline level of P-gp expression in drug-sensitive cells, which decreases the silence efficiency [56].

The current study has some limitations. First, only an appropriate US intensity, instead of a therapeutic window of US intensity, was investigated. To improve the clinical feasibility of this treatment, future study is required to explore a therapeutic window by testing additional intensity gradients. In addition, although $0.74 \mathrm{~W} / \mathrm{cm}^{2}$ US exposure didn't increase ADM concentration in apoptotic cells in peritumor muscle tissue, the effects of appropriate US 
intensity on other normal tissues should be investigated in future studies. What's more, oxidative stress induced p53 up-regulation and $\mathrm{pRb}$ de-phosphorylation are both participated in miR-200c expression, reinforcing miR-200c/ ZEB1 circuitry [30]. Whether such mechanisms also play a role in US-induced miR-200c/34a/ZEB1 feedback loop should be elucidated by future studies.

\section{Conclusions}

In conclusion, US exposure enhances ADM intracellular uptake and accumulation of MDR cancer cells in vivo and in vitro. The increased cellular uptake improved cytotoxicity in MCF-7/ADR and HEPG2/ADM cells. The anti-MDR effect of US is associated with P-gp down-regulation. US exposure increases ROS generation and activates a double feedback loop formed by miR-200c/34a/ZEB1, leading to P-gp inhibition. These findings suggest that US exposure could serve as a promising treatment for MDR. In the future, well-designed clinical studies are required to further evaluate the feasibility and efficacy of US-mediated reversal of cancer MDR.

\section{Additional files}

Additional file 1: Figure S1. Diagrammatic representation of US application in vitro. Sterilized US transducer was immersed in the culture medium and about $10 \mathrm{~mm}$ above the cell layer. (PDF $131 \mathrm{~kb}$ )

Additional file 2: Table S1. The primers used in this study. (DOCX $17 \mathrm{~kb}$ ) Additional file 3: Figure S2. (A-B) Modulation by US exposure of the sensitivity to ADM of MCF-7/ADR cells (A) and HEPG2/ADM cells (B); $N=3$, data are represented as mean \pm s.d; ${ }^{*} P<0.05$ vs. ADM group; (C) Induction of apoptosis in two MDR cells was determined by flow cytometry after treatment with US+ADM or ADM. N=3; data are represented as mean \pm s.d; ${ }^{*} P<0.05$. (PDF $265 \mathrm{~kb}$ )

Additional file 4: Table S2. The IC50 of ADM for MCF-7/ADR, HEPG2/ ADM cells and their parental cells, and HUVEC. (DOC $30 \mathrm{~kb}$ )

Additional file 5: Figure S3. The dynamic change of ROS activity in drug-resistant cells after US stimulation. (A) Representative images of DCFH-DA staining in MCF-7/ADR cells exposed to US, (scale bar $=50 \mu \mathrm{m}$ ); (B) Quantitative analysis of ROS fluorescence intensity. $N=3$; data are represented as mean \pm s.d; ${ }^{*} P<0.05$ vs. 0 h. (PDF $321 \mathrm{~kb}$ )

Additional file 6: Figure S4. MiR-200c/34a modulated MDR phenotype. (A-B) MiR-200c/24a inhibition diminished the effect of US on enhancing ADM uptake (A) and ADM-inducing apoptosis (B) for MCF-7/ADR cells; $N=3$; data are represented as mean \pm s.d; ${ }^{*} P<0.05$; (C) MiR-200c/34a overexpression increased the intracellular ADM uptake in MCF-7/ADR cells; $N=3$; data are represented as mean \pm s.d; ${ }^{*} P<0.05$; (D) MiR-200c/ 34 a overexpression increased the cytotoxicity of ADM in MCF-7/ADR cells; $N=3$; data are represented as mean $\pm \mathrm{s} . \mathrm{d} ;{ }^{*} P<0.05$; (E) TUNEL staining detected the cell apoptosis of MCF-7/ADR cells transfected with miR-200c/34a mimics or control (scale bar $=50 \mu \mathrm{m}$ ); $N=3$; data are represented as mean $\pm \mathrm{s} . \mathrm{d} ;{ }^{*} P<0.05$; (F) EdU staining detected the cell proliferation of MCF-7/ADR cells transfected with miR-200c/34a mimics or control (scale bar $=50 \mu \mathrm{m}) ; N=3$; data are represented as mean \pm s.d; ${ }^{*} P<0.05$. (PDF $332 \mathrm{~kb}$ )

Additional file 7: Figure S5. The effect of US exposure on the cytotoxicity of ADM for drug-sensitive cells. (A-B) The effects of US exposure on the sensitivity to ADM of MCF-7 cells (A) and HEPG2 cells (B); $N=3$; data are represented as mean \pm s.d; ${ }^{*} P<0.05$ vs. ADM group;
(C) Cell viability in MCF-7 and HEPG2 cells $24 \mathrm{~h}$ after US exposure with different acoustic intensities; $N=3$; ${ }^{*} P<0.05$ vs. $0 \mathrm{~W} / \mathrm{cm}^{2}$ in MCF-7 cells; ${ }^{\#} P<0.05$ vs. $0 \mathrm{~W} / \mathrm{cm}^{2}$ in HEPG2 cells; (D) Images of intracellular ADM distribution in MCF-7, HEPG2 cells $24 \mathrm{~h}$ after US+ADM treatment or ADM treatment (scale bar $=10 \mu \mathrm{m})$; $(E)$ Intracellular ADM concentration in MCF-7 and HEPG2 cells in $24 \mathrm{~h}$ after US+ADM treatment or ADM treatment; $N=3$; ${ }^{*} P<0.05$. (PDF $544 \mathrm{~kb}$ )

Additional file 8: Figure S6. US exposure has no effect on P-gp expression of MCF-7, HEPG2 and HUVEC cells. (A) Representative images and quantitative analysis of DCFH-DA staining in MCF-7, HEPG2 and HUVEC cells $24 \mathrm{~h}$ after treatment; $N=3$; ${ }^{*} P<0.05$ (scale bar $=50 \mu \mathrm{m}$ ); (B-C) Detecting P-gp expression of MCF-7 (B), HEPG2 and HUVEC cells (C) by western blotting in $24 \mathrm{~h}$ after US exposure; $N=3$; data are represented as mean \pm s.d; ${ }^{*} P<0.05$; (D) Cell morphology and cytomembrane changes in MCF-7, HEPG2 and HUVEC cells after US exposure (scale bar $=5 \mu \mathrm{m}$ ); (E) The dynamic change of ADM concentration in MCF-7, HEPG2 and HUVEC cells treated with US +ADM or ADM alone. ADM was added to the mediums in $24 \mathrm{~h}$ after exposure to US; $N=3$; data are represented as mean \pm s.d; ${ }^{*} P<0.05$; (F) The dynamic change of ADM concentration in MCF-7, HEPG 2 and HUVEC cells treated with ADM immediately after US exposure; $N=3$; data are represented as mean \pm s.d; ${ }^{*} P<0.05$. (PDF $874 \mathrm{~kb}$ )

\section{Abbreviations}

3' UTR: Three prime untranslated region; ABCB-1: ATP binding cassette subfamily B member 1; ADM: Adriamycin; CHIP: Chromatin

immunoprecipitation; DCFH-DA: Dichlorofluorescein-diacetate; FBS: Fetal bovine serum; GDMLAC: Guangdong Medical Laboratory Animal Center; HUVEC: Human umbilical vein endothelial cell; IC50: Half-inhibitory concentration; L: length; MDR: Multidrug resistance; MiRs: MicroRNAs; NAC: N-acetyl-L-cysteine; P-gp: P-glycoprotein; Q-PCR: Quantitative polymerase chain reaction; ROS: Reactive oxygen species; TF: Transcriptional factor; TUNEL: Terminal deoxynucleotidyl transferase dUTP nick end labeling; US: Ultrasound; W: width

\section{Acknowledgements}

We give our thanks to Dr. Yanmei Chen who provided useful advices on writing the manuscript.

\section{Funding}

This work was supported by grants to Jianping Bin from the National Natural Science Foundation of China (No. 81771857, No. 81571698, and No. 81271640).

\section{Availability of data and materials}

The Chip-seq datasets analyzed during the present study are available at https://www.encodeproject.org/.

\section{Authors' contributions}

$\mathrm{CH}$ designed and performed experiments; $\mathrm{SH}$ conceived experiments and wrote the manuscript. $H L, X L, B L$ and $L Z$ carried out experiments; JW, MZ, XH and $\mathrm{HZ}$ analyzed and interpreted the data; $\mathrm{XS}$ and $\mathrm{WL}$ collected the data; $\mathrm{YL}$, LY and JB designed experiments and critically revised the manuscript. All authors read and approved the final manuscript.

Ethics approval and consent to participate

The use of animals in this study was approved by the Animal Research Committee of the Southern Medical University.

Consent for publication

Not applicable.

\section{Competing interests}

The authors declare that they have no competing interest.

\section{Publisher's Note}

Springer Nature remains neutral with regard to jurisdictional claims in published maps and institutional affiliations. 


\section{Author details}

'State Key Laboratory of Organ Failure Research, Department of Cardiology, Nanfang Hospital, Southern Medical University, 1838 Guangzhou Avenue North, Guangzhou 510515, China. ${ }^{2}$ Department of Gastroenterology, Guangdong Provincial Key Laboratory of Gastroenterology, Nanfang Hospital, Southern Medical University, Guangzhou 510515, China. ${ }^{3}$ Department of Oncology, Nanfang Hospital, Southern Medical University, Guangzhou 510515, China. ${ }^{4}$ Department of Pharmacy, Nanfang Hospital, Southern Medical University, Guangzhou, People's Republic of China.

Received: 25 June 2018 Accepted: 4 September 2018 Published online: 19 September 2018

\section{References}

1. Chabner BA, Roberts TG. Chemotherapy and the war on cancer [J]. Nat Rev Cancer. 2005;5(1):65-72.

2. Kaye SB. The multidrug resistance phenotype. Brit J Cancer. 1988;58(6):691-4.

3. Longley $\mathrm{DB}$, Allen $\mathrm{WL}$, Johnston PG. Drug resistance, predictive markers and pharmacogenomics in colorectal cancer. Biochim Biophys Acta. 2006; 1766(2):184-96.

4. Alfarouk KO, Stock CM, Taylor S, Walsh M, Muddathir AK, Verduzco D, Bashir AHH, Mohammed OY, Elhassan GO, Harguindey S. Resistance to cancer chemotherapy: failure in drug response from ADME to P-gp. Cancer Cell Int. 2015;15(1):71.

5. Guertin AD, O'Neil J, Stoeck A, Reddy JA, Cristescu R, Haines BB, Hinton MC, Dorton R, Bloomfield A, Nelson M. High levels of expression of P. glycoprotein/multidrug resistance protein result in resistance to Vintafolide. Mol Cancer Ther. 2016;15(8):1998.

6. Silva R, Vilas-Boas V, Carmo H, Dinis-Oliveira RJ, Carvalho F, De LBM, Remião F. Modulation of P-glycoprotein efflux pump: induction and activation as a therapeutic strategy. Pharmacol Therapeut. 2015;149(2):1.

7. Marcoux J, Wang SC, Politis A, Ma J, Biggin PC, Zhou M, Tao H, Zhang Q, Chang G. Mass spectrometry reveals synergistic effects of nucleotides, lipids, and drugs binding to a multidrug resistance efflux pump. Pnas. 2013; 110(24):9704-9.

8. Kathawala RJ, Gupta P, Ashby CR Jr, Chen ZS. The modulation of ABC transporter-mediated multidrug resistance in cancer: a review of the past decade. Drug Resist Updat. 2015;18:1-17.

9. Kathawala RJ, Gupta P, Jr CRA, Chen ZS. The modulation of ABC transportermediated multidrug resistance in cancer: a review of the past decade. Drug Resist Update. 2015;18:1.

10. Binkhathlan Z, Lavasanifar A. P-glycoprotein inhibition as a therapeutic approach for overcoming multidrug resistance in Cancer: current status and future perspectives. Curr Cancer Drug Tar. 2013;13(3):326-46.

11. Mizutani T, Masuda M, Nakai E, Furumiya K, Togawa H, Nakamura Y, Kawai Y, Nakahira K, Shinkai S, Takahashi K. Genuine functions of P-glycoprotein (ABCB1). Curr Drug Metab. 2008;9(2):167-74.

12. Singh MS, Lamprecht A. Cargoing P-gp inhibitors via nanoparticle sensitizes tumor cells against doxorubicin. Int J Pharmaceut. 2015;478(2):745-52.

13. Abbasi M, Lavasanifar A, Uludag H. Recent attempts at RNAi-mediated Pglycoprotein downregulation for reversal of multidrug resistance in cancer. Med Res Rev. 2013;33(1):33-53.

14. Yang T, Zheng ZM, Li XN, Li ZF, Wang Y, Geng YF, Bai L, Zhang XB. MiR-223 modulates multidrug resistance via downregulation of $A B C B 1$ in hepatocellular carcinoma cells. Exp Biol Med. 2013;238(9):1024.

15. Zhaolin C, Taotao M, Cheng H, Lei Z, Xiongwen L, Tao X, Tingting H, Jun L. MiR-27a modulates the MDR1 /P-glycoprotein expression by inhibiting FZD7/ $\beta$-catenin pathway in hepatocellular carcinoma cells. Cell Signal. 2013; 25(12):2693-701.

16. Yu H, Xu L. Cell experimental studies on sonoporation: state of the art and remaining problems. J Control Release. 2014;174(1):151.

17. Wu F, Shao ZY, Zhai BJ, Zhao CL, Shen DM. Ultrasound reverses multidrug resistance in human Cancer cells by altering gene expression of $A B C$ transporter proteins and Bax protein. Ultrasound Med Biol. 2011;37(1):151-9.

18. Aryal M, Fischer K, Gentile C, Gitto S, Zhang YZ, Mcdannold N. Effects on Pglycoprotein expression after blood-brain barrier disruption using focused ultrasound and microbubbles. PLoS One. 2017;12(1):e166061.

19. Cho HS, Lee HY, Han M, Choi J, Ahn S, Lee T, Chang Y, Park J. Localized Down-regulation of P-glycoprotein by focused ultrasound and microbubbles induced blood-brain barrier disruption in rat brain. Sci Rep. 2016;6:31201.
20. Liang Y, Liu Z, Guo S. Ultrasound reverses adriamycin-resistance in nonsmall cell lung cancer via positive regulation of BRAF-activated non-coding RNA (BANCR) expression. Clin Surg Res Commun. 2017;1(1):18-23.

21. Zhang Z, Xu K, Bi Y, Yu G, Wang S, Qi X, Zhong H. Low intensity ultrasound promotes the sensitivity of rat brain glioma to doxorubicin by Downregulating the expressions of P-Glucoprotein and multidrug resistance protein 1 In Vitro and In Vivo. PLoS One. 2013;8(8):e70685.

22. Deng Z, Yan F, Jin Q, Li F, Wu J, Liu X, Zheng H. Reversal of multidrug resistance phenotype in human breast cancer cells using doxorubicinliposome-microbubble complexes assisted by ultrasound. J Control Release. 2014;174(1):109-16.

23. Xu X, Yi Z, Dan Q, Jiang T, Li S. Osthole induces G2/M arrest and apoptosis in lung cancer A549 cells by modulating PI3K/Akt pathway. J Exp Clin Canc Res. 2011;30(1):33.

24. Cai H, Kapoor A, He R, Venkatadri R, Forman M, Posner GH, Arav-Boger R. In vitro combination of anti-cytomegalovirus compounds acting through different targets: role of the slope parameter and insights into mechanisms of action. Antimicrob Agents Chemother. 2014;58(2):986-94.

25. Jang KW, Ding L, Seol D, Lim TH, Buckwalter JA, Martin JA. Low-intensity pulsed ultrasound promotes chondrogenic progenitor cell migration via focal adhesion kinase pathway. Ultrasound Med Biol. 2014;40(6):1177-86.

26. Ma JX, Jin ZD, Si PR, Liu Y, Lu Z, Wu HY, Pan X, Wang LW, Gong YF, Gao J. Continuous and low-energy 125 I seed irradiation changes DNA methyltransferases expression patterns and inhibits pancreatic cancer tumor growth. J Exp Clin Canc Res. 2011;30(1):35.

27. Surowiak P, Materna V, Matkowski R, Szczuraszek K, Kornafel J, Wojnar A Pudelko M, Dietel M, Denkert C, Zabel M. Relationship between expression of cyclooxygenase 2 and MDR1/P-glycoprotein in invasive breast cancers and their prognostic significance. Breast Cancer Res. 2005;7(5):R862-70.

28. Jaksik R, Lalik A, Skonieczna M, Cieslar-Pobuda A, Student S, RzeszowskaWolny J. MicroRNAs and reactive oxygen species: are they in the same regulatory circuit? Mutat Res Genet Toxicol Environ Mutagen. 2014;764765(4):64-71.

29. Sui H, Cai GX, Pan SF, Deng WL, Wang YW, Chen ZS, Cai SJ, Zhu HR, Li Q. miR200C attenuates P-gp-mediated MDR and metastasis by targeting JNK2/CJun signaling pathway in colorectal cancer. Mol Cancer Ther. 2014:13(12):3137.

30. Magenta A, Cencioni C, Fasanaro P, Zaccagnini G, Greco S, Sarra-Ferraris G, Antonini A, Martelli F, Capogrossi MC. miR-200c is upregulated by oxidative stress and induces endothelial cell apoptosis and senescence via ZEB1 inhibition. Cell Death Differ. 2011;18(10):1628.

31. Rajabi H, Alam M, Takahashi H, Kharbanda A, Guha M, Ahmad R, Kufe D. MUC1-C oncoprotein activates the ZEB1/miR-200c regulatory loop and epithelial-mesenchymal transition. Oncogene. 2014;33(13):1680-9.

32. Sun $Y, L i Q, X u Y, P u C$, Zhao $L$, Guo Z, Ding $X$, Jin $X$. Study of the mechanisms underlying the reversal of multidrug resistance of human neuroblastoma multidrug-resistant cell line SK-N-SH/MDR1 by low-intensity pulsed ultrasound. Oncol Rep. 2013;29(5):1939-45.

33. Yu T, Bai J, Hu K, Wang Z. Biological effects of ultrasound exposure on adriamycin-resistant and cisplatin-resistant human ovarian carcinoma cell lines in vitro. Ultrason Sonochem. 2004;11(2):89-94.

34. Yu H, Xu L, Chen S. A transfer efficiency model for ultrasound mediated drug/gene transferring into cells. Ultrason Sonochem. 2014;21(1):113-20.

35. Szakács G, Paterson JK, Ludwig JA, BoothGenthe C, Gottesman MM. Targeting multidrug resistance in cancer. Nat Rev Drug Discov. 2006;5(3):219-34.

36. Nieth $C$, Lage $H$. Induction of the ABC-transporters Mdr1/P-gp (Abcb1), mrpl (Abcc1), and bcrp (Abcg2) during establishment of multidrug resistance following exposure to mitoxantrone. J Chemother. 2005;17(2):215-23.

37. Furusawa Y, Zhao QL, Hassan MA, Tabuchi Y, Takasaki I, Wada S, Kondo T. Ultrasound-induced apoptosis in the presence of Sonazoid and associated alterations in gene expression levels: a possible therapeutic application. Cancer Lett. 2010;288(1):107-15.

38. Terada Y, Ogura J, Tsujimoto T, Kuwayama K, Koizumi T, Sasaki S, Maruyama $\mathrm{H}$, Kobayashi M, Yamaguchi H, Iseki K. Intestinal P-glycoprotein expression is multimodally regulated by intestinal ischemia-reperfusion. J Pharm Pharm Sci. 2014;17(2):266-76.

39. Wartenberg M, Richter M, Datchev A, Günther S, Milosevic N, Bekhite MM, Figulla HR, Aran JM, Pétriz J, Sauer H. Glycolytic pyruvate regulates Pglycoprotein expression in multicellular tumor spheroids via modulation of the intracellular redox state. J Cell Biochem. 2010;109(2):434-46.

40. Maria W, Frederike CL, Markus M, Florian K, Helmut A, Max G, Kerstin P, Volker $P$, Jürgen $H$, Heinrich $S$. Regulation of the multidrug resistance 
transporter P-glycoprotein in multicellular tumor spheroids by hypoxiainducible factor-1 and reactive oxygen species. FASEB J. 2003;17(3):503.

41. Wartenberg M, Hoffmann E, Schwindt H, Grünheck F, Petros J, Arnold JR, Hescheler J, Sauer $\mathrm{H}$. Reactive oxygen species-linked regulation of the multidrug resistance transporter P-glycoprotein in Nox-1 overexpressing prostate tumor spheroids. FEBS Lett. 2005;579(20):4541-9.

42. Duco W, Grosso V, Zaccari D, Soltermann AT. Generation of ROS mediated by mechanical waves (ultrasound) and its possible applications. Methods. 2016;109:141.

43. Magenta A, Greco S, Gaetano C, Martelli F. Oxidative stress and microRNAs in vascular diseases. Int J Mol Sci. 2013;14(9):17319-46.

44. Cheleschi S, Palma AD, Pascarelli NA, Giordano N, Galeazzi M, Tenti S, Fioravanti A. Could oxidative stress regulate the expression of MicroRNA146a and MicroRNA-34a in human osteoarthritic chondrocyte cultures? Int J Mol Sci. 2017:18(12):2660

45. Li ZH, Weng X, Xiong QY, Tu JH, Xiao A, Qiu W, Gong Y, Hu EW, Huang S, Cao YL. miR-34a expression in human breast cancer is associated with drug resistance. Oncotarget. 2017;8(63):106270-82.

46. Sun C, Wang F-J, Zhang H-G, XU X-Z, Jia R-C, Yao L, Qiao P-F. miR-34a mediates oxaliplatin resistance of colorectal cancer cells by inhibiting macroautophagy via transforming growth factor- $\beta / S$ mad4 pathway. World J Gastroentero. 2017:23(10):1816-27.

47. Ng WL, Chen G, Wang M, Wang H, Story M, Shay JW, Zhang X, Wang J, Amin AR, Hu B, Cucinotta FA, Wang Y. OCT4 as a target of miR-34a stimulates p63 but inhibits p53 to promote human cell transformation. Cell Death Dis. 2014;5(1):e1024

48. Li D, Jian L. Association of miR-34a-3p/5p, miR-141-3p/5p, and miR-24 in Decidual natural killer cells with unexplained recurrent spontaneous abortion. Med Sci Monit. 2016;22:922-9.

49. Guennewig B, Roos M, Dogar AM, Gebert LFR, Zagalak JA, Vongrad V, Metzner KJ, Hall J. Synthetic pre-microRNAs reveal dual-strand activity of miR-34a on TNF-a. RNA. 2014;20(1):61.

50. Chen J, Tian W, Cai H, He H, Deng Y. Down-regulation of microRNA-200C is associated with drug resistance in human breast cancer. Med Oncol. 2012; 29(4):2527-34

51. Espinosadiez C, Miguel V, Mennerich D, Kietzmann T, Sánchezpérez $P$, Cadenas S, Lamas S. Antioxidant responses and cellular adjustments to oxidative stress. Redox Biol. 2015;6:183-97.

52. Chen Z, Shentu TP, Wen L, Johnson DA, Shyy JY. Regulation of SIRT1 by oxidative stress-responsive miRNAs and a systematic approach to identify its role in the endothelium. Antioxid Redox Sign. 2013;19(13):1522-38.

53. Burk U, Schubert J, Wellner U, Schmalhofer O, Vincan E, Spaderna S, Brabletz T. A reciprocal repression between $Z E B 1$ and members of the miR-200 family promotes EMT and invasion in cancer cells. EMBO Rep. 2008;9(6):582-9.

54. Ahn YH, Gibbons DL, Chakravarti D, Creighton CJ, Rizvi ZH, Adams HP, Pertsemlidis A, Gregory PA, Wright JA, Goodall GJ. ZEB1 drives prometastatic actin cytoskeletal remodeling by downregulating miR-34a expression. J Clin Invest. 2012:122(9):3170-83.

55. Zhang J, Du Z, Pan S, Shi M, Li J, Yang C, Hu H, Qiao M, Chen D, Zhao X. Overcoming multidrug resistance by Codelivery of MDR1-targeting siRNA and doxorubicin using EphA10-mediated pH-sensitive Lipoplexes: In Vitro and In Vivo evaluation. ACS Appl Mater Inter. 2018;10(25):21590-600.

56. Yuan WQ, Zhang RR, Wang J, Ma Y, Li WX, Jiang RW, Cai SH. Asclepiasterol, a novel C21 steroidal glycoside derived from Asclepias curassavica, reverses tumor multidrug resistance by down-regulating P-glycoprotein expression. Oncotarget. 2016;7(21):31466-83.

Ready to submit your research? Choose BMC and benefit from:

- fast, convenient online submission

- thorough peer review by experienced researchers in your field

- rapid publication on acceptance

- support for research data, including large and complex data types

- gold Open Access which fosters wider collaboration and increased citations

- maximum visibility for your research: over $100 \mathrm{M}$ website views per year

At BMC, research is always in progress.

Learn more biomedcentral.com/submissions 